\title{
An Application of Multi-band Forced Photometry to One Square Degree of SERVS: Accurate Photometric Redshifts and Implications for Future Science
}

\author{
Kristina Nyland ${ }^{1}$, Mark Lacy ${ }^{1}$, Anna Sajina ${ }^{2}$, Janine Pforr ${ }^{3,4}$, Duncan Farrah ${ }^{5}$, Gillian Wilson ${ }^{6}$, Jason Surace ${ }^{7}$, Boris Häußler ${ }^{8}$, \\ Mattia Vaccari ${ }^{9,10}$, and Matt Jarvis ${ }^{11,12}$ \\ ${ }^{1}$ National Radio Astronomy Observatory, Charlottesville, VA 22903, USA; knyland@nrao.edu \\ ${ }^{2}$ Department of Physics \& Astronomy, Tufts University, Medford, MA 02155, USA \\ ${ }^{3}$ ESA/ESTEC SCI-S, Keplerlaan 1, 2201 AZ, Noordwijk, The Netherlands \\ ${ }^{4}$ Aix Marseille Université, CNRS, LAM (Laboratoire d'Astrophysique de Marseille) UMR 7326, F-13388, Marseille, France \\ 5 Department of Physics, Virginia Tech, Blacksburg, VA 24061, USA \\ ${ }^{6}$ Department of Physics and Astronomy, University of California-Riverside, 900 University Avenue, Riverside, CA, 92521, USA \\ ${ }_{7}^{7}$ Spitzer Science Center, California Institute of Technology, M/S 314-6, Pasadena, CA 91125, USA \\ ${ }^{8}$ European Southern Observatory, Alonso de Cordova 3107, Vitacura, Casilla 19001, Santiago, Chile \\ ${ }^{9}$ Department of Physics and Astronomy, University of the Western Cape, Robert Sobukwe Road, 7535 Bellville, Cape Town, South Africa \\ ${ }^{10}$ INAF-Istituto di Radioastronomia, via Gobetti 101, I-40129 Bologna, Italy \\ ${ }^{11}$ Department of Physics, Oxford Astrophysics, University of Oxford, Keble Road, Oxford OX1 3RH, UK \\ ${ }^{12}$ Physics Department, University of the Western Cape, Cape Town 7535, South Africa \\ Received 2016 December 30; revised 2017 April 4; accepted 2017 April 11; published 2017 May 24
}

\begin{abstract}
We apply The Tractor image modeling code to improve upon existing multi-band photometry for the Spitzer Extragalactic Representative Volume Survey (SERVS). SERVS consists of post-cryogenic Spitzer observations at 3.6 and $4.5 \mu \mathrm{m}$ over five well-studied deep fields spanning $18 \mathrm{deg}^{2}$. In concert with data from ground-based near-infrared (NIR) and optical surveys, SERVS aims to provide a census of the properties of massive galaxies out to $z \approx 5$. To accomplish this, we are using The Tractor to perform "forced photometry." This technique employs prior measurements of source positions and surface brightness profiles from a high-resolution fiducial band from the VISTA Deep Extragalactic Observations survey to model and fit the fluxes at lower-resolution bands. We discuss our implementation of The Tractor over a square-degree test region within the XMM Large Scale Structure field with deep imaging in $12 \mathrm{NIR} /$ optical bands. Our new multi-band source catalogs offer a number of advantages over traditional position-matched catalogs, including (1) consistent source cross-identification between bands, (2) deblending of sources that are clearly resolved in the fiducial band but blended in the lower resolution SERVS data, (3) a higher source detection fraction in each band, (4) a larger number of candidate galaxies in the redshift range $5<z<6$, and (5) a statistically significant improvement in the photometric redshift accuracy as evidenced by the significant decrease in the fraction of outliers compared to spectroscopic redshifts. Thus, forced photometry using The Tractor offers a means of improving the accuracy of multi-band extragalactic surveys designed for galaxy evolution studies. We will extend our application of this technique to the full SERVS footprint in the future.
\end{abstract}

Key words: catalogs - Galaxies: evolution - methods: data analysis - surveys - techniques: image processing

Supporting material: machine-readable table

\section{Introduction and Motivation}

Beginning with the successful SIRTF Wide-area Infrared Extragalactic Legacy Survey (SWIRE; Lonsdale et al. 2003) over a decade ago, a growing number of deep extragalactic surveys with the Spitzer Space Telescope (Werner et al. 2004) have provided unprecedented insights into the evolution of galaxies over cosmic time. Although the SWIRE footprint spanned $\sim 49 \mathrm{deg}^{2}$ and included imaging in seven Spitzer bands from the near to far infrared, it was primarily sensitive to objects at low and intermediate redshifts of $z \lesssim 3$ due to its relatively shallow depth. More recently, the abundance of postcryogenic observing time with Spitzer for large programs has made the construction of wide-area deep fields more feasible in the shortest two bands of the Infrared Array Camera (IRAC; Fazio et al. 2004) that have remained in operation. This has been particularly fortuitous for galaxy evolution studies since the combination of the inherent shapes of galaxy spectral energy distributions (SEDs) and the low background level in the IRAC bands make IRAC uniquely well equipped for the detection of high-redshift galaxies given sufficient exposure time (e.g., Oesch et al. 2014). Furthermore, until the launch of the James Webb Space Telescope (JWST), currently scheduled for late 2018, IRAC is one of the few instruments capable of detecting rest-frame optical emission from galaxies at $z>4$.

A number of recent surveys have capitalized on the opportunity to conduct deep, wide-area IRAC surveys at 3.6 and $4.5 \mu \mathrm{m}$ during the warm phase of the Spitzer mission capable of detecting high-redshift galaxies. Examples include the Spitzer IRAC Equatorial Survey (SpIES; Timlin et al. 2016), the Spitzer-HETDEX Exploratory Large-Area Survey (Papovich et al. 2016), and the Spitzer Extragalactic Representative Volume Survey (SERVS; Mauduit et al. 2012). Here, we focus on the SERVS project, which is deep enough to detect $L_{*}$ galaxies at $z \approx 5$, but still wide enough to detect interesting and rare objects such as quasars and ultraluminous infrared galaxies. Thus, in concert with abundant ancillary data that include deep observations at optical, far-infrared, and radio wavelengths, SERVS will provide new insights into the cosmic star formation and supermassive black hole accretion histories of galaxies over the redshift range of $z \sim 0-5$. 
The basis for the scientific success of surveys such as SERVS lies in the construction of robust multi-band source catalogs. The existing SERVS source catalogs (Mauduit et al. 2012) were constructed using traditional photometric methods and software (e.g., SExtractor; Bertin \& Arnouts 1996). These methods typically employ an aperture photometry approach, in which fluxes are computed within a fixed elliptical aperture. This technique generally yields singleband source catalogs of acceptable accuracy. However, the suite of multi-wavelength SERVS data incorporate ancillary ground-based NIR and optical imaging at higher spatial resolution (0!.8) compared to that of the IRAC 3.6 and $4.5 \mu \mathrm{m}$ bands ( 1 ". 95 and 2 ". 02 , respectively). Mixed-resolution, multi-band catalogs are typically constructed by performing positional cross-matching between the individual source catalogs for each band within a predefined search radius (e.g., Vaccari 2015). A drawback to this approach for SERVS is that sources that are clearly resolved in the higher resolution, ground-based bands may appear "blended" together as a single source in the Spitzer IRAC imaging. If not corrected, this will inevitably lead to incorrect source cross-identification between bands as well as less accurate flux measurements and photometric redshifts.

In response to the need for more accurate multi-band photometry, a number of new tools have been developed in recent years. These include software packages that use prior information from a band with high-resolution imaging to model the flux in lower-resolution bands such as T-PHOT (the successor to TFIT; Merlin et al. 2015, 2016b), PyGFIT (Mancone et al. 2013), XID+ (Hurley et al. 2017), and other applications of Bayesian cross-matching (Marquez et al. 2014; Budavári \& Basu 2016), LAMDAR (Wright et al. 2016), and The Tractor (Lang et al. 2016a, 2016b). Each of these tools has different strengths and weaknesses in areas such as the available options for modeling sources (point-source/resolved surface brightness profile versus elliptical aperture), fitting heuristics (maximum likelihood estimator versus Bayesian inference), point-spread function (PSF) characterization (single Gaussian/mixture of Gaussians versus model image), algorithm speed, and accessibility to the user community.

T-PHOT, PyGFIT, and The Tractor offer source surface brightness profile modeling capabilities, an important feature for producing accurate multi-band photometry of resolved sources in crowded, mixed-resolution data sets. The most widely used software that includes analytic source modeling as an option is T-PHOT (e.g., Merlin et al. 2016a). However, T-PHOT has a number of stringent image formatting requirements, such as the need for perfectly aligned pixel boundaries between the lowresolution and high-resolution images. This complicates matters for users who wish to perform multi-band photometry using source catalogs and images from surveys with heterogeneous sky footprints. In addition, analytic surface brightness profile modeling in both T-PHOT and PyGFIT requires users to perform an additional step of producing a model image based on the highest-resolution band using software such as the GALFIT (Peng et al. 2002), thus increasing the computation time. In contrast to T-PHOT and PyGFIT, The Tractor provides a greater degree of flexibility, simplicity, and customization opportunities (for details, see Section 3 and Lang et al. 2016a). Thus, The Tractor is well suited to the application of multi-band optical/NIR photometry considered in this study.
Here, we present new multi-band forced photometry incorporating data from 12 NIR and optical bands over a square degree of the XMM Large Scale Structure (XMM-LSS) field included in SERVS. We describe the SERVS project in detail in Section 2 and the heuristics of our application of forced photometry using The Tractor in Section 3. In Section 4, we compare the basic properties of our new multi-band forced photometric catalogs with the original input source catalogs constructed using traditional positional matching between bands. We discuss the color and photometric redshift accuracy of our new forced-photometry catalogs and also consider prospects for future science applications in Section 5. We summarize our results in Section 6. Throughout this study we adopt a $\Lambda$ CDM cosmology with $\Omega_{\mathrm{M}}=0.3, \Omega_{\Lambda}=0.7$, and $H_{0}=70 \mathrm{~km} \mathrm{~s}^{-1} \mathrm{Mpc}^{-1}$.

\section{SERVS}

\subsection{Overview}

The SERVS sky footprint includes five well-studied astronomical deep fields with abundant multi-wavelength data spanning an area of $\approx 18 \mathrm{deg}^{2}$ and a co-moving volume of $\approx 0.8 \mathrm{Gpc}^{3}$. The five deep fields included in SERVS are the $X M M$-LSS field, Lockman Hole (LH), ELAIS-N1 (EN1), ELAIS-S1 (ES1), and Chandra Deep Field South (CDFS). SERVS provides NIR, post-cryogenic imaging in the 3.6 and $4.5 \mu \mathrm{m}$ IRAC bands to a depth of $\approx 2 \mu \mathrm{Jy}$. IRAC dual-band source catalogs generated using traditional catalog extraction methods are described in Mauduit et al. (2012).

The Spitzer IRAC data are complemented by ground-based NIR observations from the VISTA Deep Extragalactic Observations (VIDEO; Jarvis et al. 2013) survey in the south in the $Z, Y, J, H$, and $K_{\mathrm{s}}$ bands and UKIRT Infrared Deep Sky Survey (UKIDSS; Lawrence et al. 2007) in the north in the $J$ and $K$ bands. SERVS also provides substantial overlap with infrared data from SWIRE (Lonsdale et al. 2003) and the Herschel Multitiered Extragalactic Survey (HerMES; Oliver et al. 2012).

Multi-band "data fusion" source catalogs for all five SERVS fields combining data from spectroscopic redshift surveys and photometry from the far-ultraviolet to the far-infrared using standard position-matching are described in Vaccari (2015). The SERVS data fusion multi-band catalogs are based on the IRAC 3.6 and $4.5 \mu \mathrm{m}$ band-merged catalog presented in Mauduit et al. (2012). For each SERVS source, the IRAC position is cross-matched with existing multi-wavelength source catalogs within a search radius of $1^{\prime \prime}$. For further details on the construction and contents of the SERVS data fusion catalogs, we refer readers to Vaccari (2015).

The suite of multiwavlength data available in the SERVS fields at NIR and optical wavelengths are especially well suited for determining photometric redshifts for high-redshift objects (e.g., Ilbert et al. 2009). Photometric redshifts over the five SERVS fields based on the data fusion catalogs will be presented in J. Pforr et al. (2017, in preparation).

\subsection{Square-degree Test Field}

As shown in Figure 1, one square degree of the XMM-LSS field overlaps with ground-based optical data from the Canada-FranceHawaii Telescope Legacy Survey Deep field 1 (CFHTLS-D1). The CFHTLS-D1 region is centered at R.A.(J2000) = 02:25:59, decl.(J2000) $=-04: 29: 40$ and includes imaging through the filter 


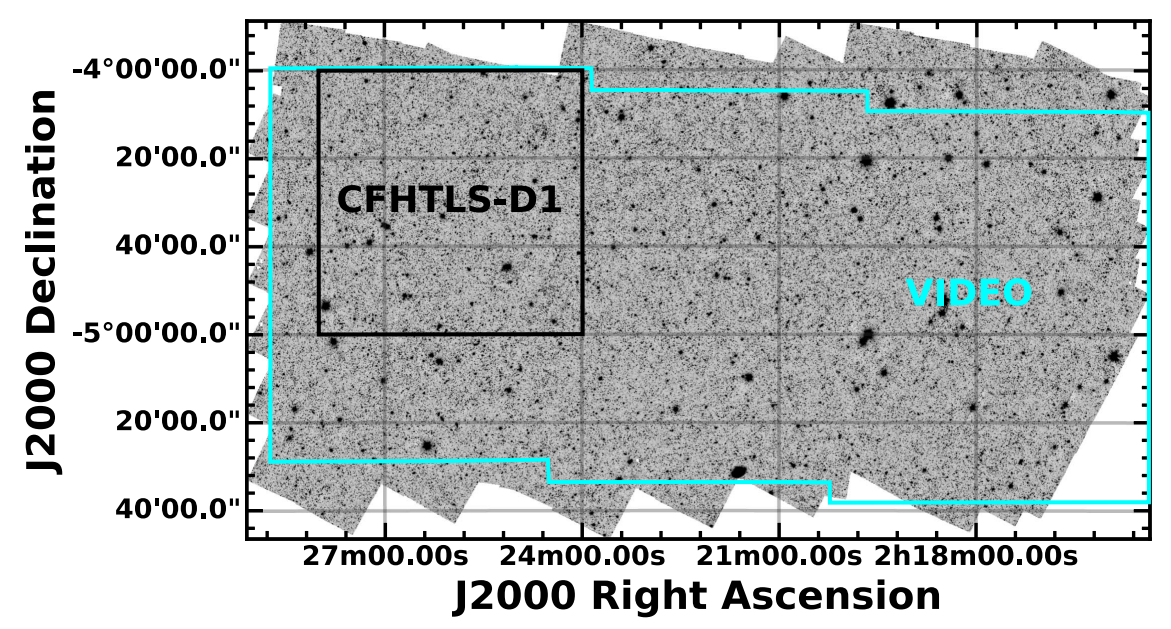

Figure 1. Spitzer IRAC $3.6 \mu \mathrm{m}$ image from SERVS (Mauduit et al. 2012) of the XMM-LSS field. The cyan polygon traces the footprint of the VIDEO survey (Jarvis et al. 2013). The black square region denotes the location of the CFHTLS-D1 square-degree footprint (Gwyn 2012) over which we have tested the implementation of forced photometry with The Tractor.

Table 1

Summary of Multi-band Data in the XMM-LSS Square-degree Test Field

\begin{tabular}{|c|c|c|c|c|c|}
\hline $\begin{array}{l}\text { Band } \\
\text { (1) }\end{array}$ & $\begin{array}{l}\text { Telescope } \\
\text { (2) }\end{array}$ & $\begin{array}{l}\text { Survey } \\
\text { (3) }\end{array}$ & $\begin{array}{l}\lambda(\mu \mathrm{m}) \\
\quad(4)\end{array}$ & $\begin{array}{c}5 \sigma \text { Threshold } \\
\text { (5) }\end{array}$ & $\begin{array}{c}\text { Angular Resolution (arcsec) } \\
\text { (6) }\end{array}$ \\
\hline \multicolumn{6}{|c|}{ Near Infrared } \\
\hline [4.5] & Spitzer & SERVS & 4.50 & 23.1 & 2.02 \\
\hline [3.6] & Spitzer & SERVS & 3.60 & 23.1 & 1.95 \\
\hline$K_{\mathrm{s}}$ & VISTA & VIDEO & 2.15 & 23.8 & 0.84 \\
\hline$H$ & VISTA & VIDEO & 1.65 & 24.1 & 0.88 \\
\hline$J$ & VISTA & VIDEO & 1.25 & 24.4 & 0.87 \\
\hline$Y$ & VISTA & VIDEO & 1.02 & 24.5 & 0.86 \\
\hline$Z$ & VISTA & VIDEO & 0.88 & 25.7 & 0.88 \\
\hline \multicolumn{6}{|c|}{ Optical } \\
\hline$z^{\prime}$ & CFHT & CFHTLS-D1 & 0.93 & 26.2 & 0.81 \\
\hline$i^{\prime}$ & CFHT & CFHTLS-D1 & 0.78 & 27.4 & 0.76 \\
\hline$r^{\prime}$ & CFHT & CFHTLS-D1 & 0.64 & 27.7 & 0.77 \\
\hline$g^{\prime}$ & CFHT & CFHTLS-D1 & 0.47 & 27.9 & 0.83 \\
\hline$u^{\prime}$ & CFHT & CFHTLS-D1 & 0.35 & 27.5 & 0.87 \\
\hline
\end{tabular}

Note. Column 1: observing band or filter name. Column 2: telescope name. Column 3: survey name. Column 4: central wavelength of observing band/filter. Column 5: $5 \sigma$ source detection threshold. Thresholds are taken from Mauduit et al. (2012) for SERVS, Jarvis et al. (2013) for VIDEO (2" aperture), and Gwyn (2012) for CFHTLS-D1. All measurements are in AB magnitudes. Column 6: typical angular resolution in the final survey images. For the ground-based VIDEO and CFHTLSD1 observations, the angular resolution refers to the typical seeing conditions. For the SERVS data, the angular resolution refers to the FWHM of the post-cryogenic IRAC PRF from the IRAC Instrument Handbook.

set $u^{\prime}, g^{\prime}, r^{\prime}, i^{\prime}$, and $z^{\prime}$. Thus, in combination with the NIR data from SERVS and VIDEO that overlap with the CFHTLS-D1 region, multi-band imaging over a total of 12 bands is available (Table 1). Because of the abundant multi-band data in the CFHTLS-D1 field, we use this field to test our implementation of multi-band photometry with The Tractor.

\section{Method}

\subsection{The Tractor}

The Tractor (Lang et al. 2016a, 2016b) uses prior source positions, fluxes, and shape information from a high-resolution, ground-based NIR fiducial band to model and fit the flux in the remaining NIR and optical bands. Fitting using The Tractor essentially optimizes the likelihood for the photometric properties of each source in each band given initial information on the source and image parameters. Input image parameters for each band include a noise model, a PSF model, image astrometric information (WCS), and calibration information (e.g., the "sky noise" or rms of the image background). The input source parameters include the source positions, brightnesses, and surface brightness profile shapes. The Tractor proceeds by rendering the source model convolved with the image PSF model at each band and performs a least-squares fit to the image data. Since The Tractor is made freely available to the astronomical user community in the form of a PYTHON module, it does not have a front-end user interface and users must write a driver script.

\subsection{Input Catalogs}

The Tractor must be supplied with an input catalog of prior positions. To construct our input catalog, we first cross-matched 


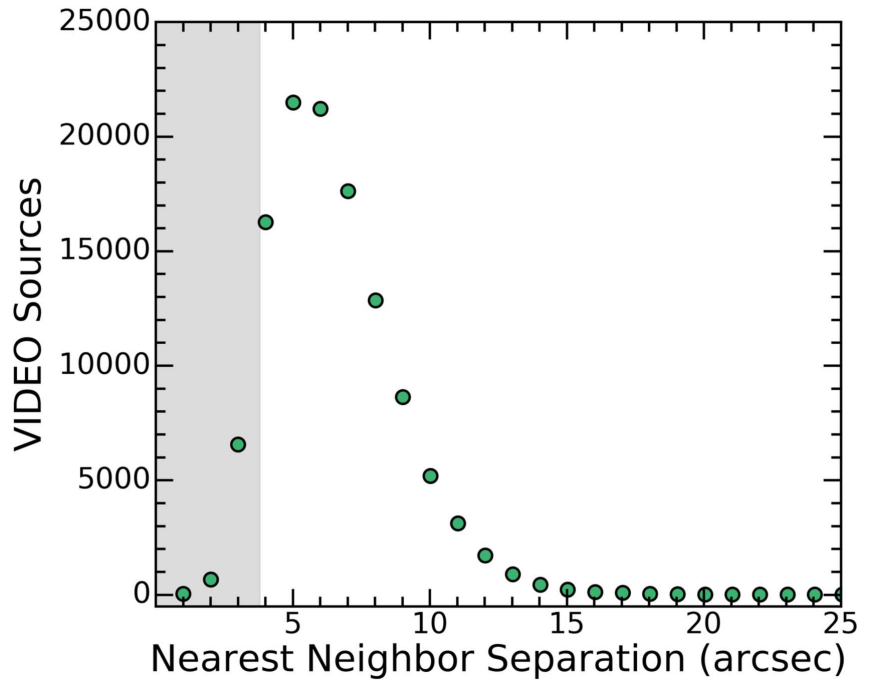

Figure 2. Number distribution of nearest-neighbor VIDEO catalog source separations $(\Delta \theta)$. The gray-shaded portion highlights the population of VIDEO sources with $\Delta \theta<3$. $^{\prime \prime}$ that are expected to be blended in the original catalog of SERVS IRAC aperture photometry within a radius of 1.19 , which corresponds to the FLUX_APER_2_1 and FLUX_APER_2_2 apertures for the 3.6 and $4.5 \mu \mathrm{m}$ bands, respectively.

the VIDEO ${ }^{13}$ and SERVS catalogs using TOPCAT (Taylor 2005), retaining all VIDEO sources as well as any SERVS source matches within a search radius of $1^{\prime \prime}$ in our test field. We then matched this VIDEO-SERVS catalog with the ground-based optical CFHTLS-D1 catalog, again retaining all VIDEO sources and any CFHTLS-D1 matches within a search radius of $1^{\prime \prime}$. The resulting VIDEO-selected, multi-band input catalog contains 12 NIR and optical bands (see Table 1) and a total of 117,281 objects.

Based on the number distribution of nearest-neighbor source separations of $\Delta \theta \lesssim 3$ ". 8 (or about twice the angular resolution of the SERVS data) shown in Figure 2, we expect at least $17 \%$ of the 117,281 sources in the VIDEO-selected input catalog will be blended in the 3.6 and $4.5 \mu \mathrm{m}$ IRAC data. This is a lower limit since VIDEO sources with larger intrinsic angular sizes will be blended on even larger spatial scales in the original, position-matched SERVS photometric catalogs. The high fraction of VIDEO sources expected to be blended in SERVS is one of the primary motivations for performing forced photometry with The Tractor.

To avoid biasing our output catalog against faint or extremely red objects that are detected only in the IRAC bands and have no ground-based NIR or optical counterparts, we also created a secondary input catalog of IRAC-selected sources. ${ }^{14}$ For this catalog, we included all detected SERVS sources lacking a counterpart within $1^{\prime \prime}$ in the original VIDEO source catalog. We also required a detection in at least one of the two IRAC bands in the SERVS single-band 3.6 and $4.5 \mu \mathrm{m}$ catalogs. ${ }^{15}$ The resulting IRAC-selected input catalog contains 8441 sources.

\footnotetext{
13 VIDEO source catalogs and images were obtained from the fourth data release available at http://horus.roe.ac.uk/vsa/. All VIDEO data presented in this study are based on the VIDEO DR4.

${ }^{14}$ We emphasize that we perform forced photometry using The Tractor on both the VIDEO-selected and IRAC-selected input catalogs, thus producing two separate output multi-band source catalogs.

${ }^{15}$ http://irsa.ipac.caltech.edu/data/SPITZER/SERVS/
}

\subsection{Fiducial Band Selection}

The Tractor generates a user-defined model of the surface brightness profile (see Section 3.4) of a source at a given "fiducial" band with high-spatial-resolution imaging and then convolves this model with the PSF of each remaining, lowerresolution band. Thus, the first step in our source modeling procedure is to determine the fiducial band. For the VIDEOselected catalog, we use the VIDEO $K_{\mathrm{s}}$-band data to define the fiducial high-resolution model of each source when possible since this band is closest in wavelength to the IRAC bands. However, for sources in the VIDEO-selected catalog with nondetections at $K_{\mathrm{s}}$ band, we select a fiducial VIDEO band with a detection and valid flux entry with a preference for the band with the next closest central wavelength to the $3.6 \mu \mathrm{m}$ IRAC data. We note that, while we could select an optical band from the CFHTLS-D1 data as the fiducial band, this might result in the loss of very red objects from the catalog and the possible misappropriation of infrared flux to unrelated galaxies with blue optical-infrared colors.

We follow a similar strategy for the IRAC-selected catalog of red sources detected in at least one IRAC band that lack a counterpart detected in any of the VIDEO bands in the original VIDEO catalog. If a source is only detected in a single IRAC band, then that band is the fiducial band. However, if both the 3.6 and $4.5 \mu \mathrm{m}$ bands are detected, then we select the $3.6 \mu \mathrm{m}$ band as the fiducial band. The fiducial band selected for each source is provided in the Fiducial_Band column (see Table 4 in the Appendix) of both the VIDEO- and IRAC-selected output catalogs.

\subsection{Surface Brightness Profile Modeling}

Once the fiducial band has been determined, we extract an image cutout of each source in the input VIDEO-selected catalog from the mosaicked image at each band using the PYTHON wrapper to the MONTAGE ${ }^{16}$ toolkit (Berriman \& Good 2017), which is able to robustly interpret the complex WCS information in the headers of the Spitzer IRAC mosaics. The resulting image cutouts each have a half-width of $5^{\prime \prime}$. This cutout size represents a trade-off between ensuring that the sources in our test field lie well within the cutout extent and excessive computational costs associated with larger cutout sizes. Next, we create a fiducial-band model of the target object as well as any neighboring sources in the VIDEO-selected input catalog that are present in the image cutout.

Based on the fiducial-band image, the source of interest along with neighboring sources within the cutout are modeled as either unresolved (i.e., a point source) or resolved. For a source to be considered resolved, we require it to have a low probability of being a star in the VIDEO catalog (PSTAR $<0.1$ ) and an estimated radius $r>0$ !" 1 . The radius is defined as $r_{\text {source }}=\theta_{\text {maj }} \times \sqrt{b / a}+0.1$, where $\theta_{\text {maj }}$ is the seeing-corrected half-light, semimajor axis (KSHLCORSMJRADAS for $K_{\mathrm{s}}$ band in the original VIDEO source catalog), $b / a$ is the axis ratio (semiminor/semimajor) of an ellipse describing the source extent (determined from the KSELL VIDEO catalog parameter, where $b / a=1-\mathrm{KSELL}$ ), and the constant 0.1 refers to half the pixel size $\left(0{ }^{\prime \prime} 2\right)$ the VIDEO bands.

Photometry for resolved sources is then performed twiceonce using a deVaucouleurs profile (equivalent to a Sérsic

\footnotetext{
${ }^{16}$ http://montage.ipac.caltech.edu
} 
Table 2

Summary of Image Calibration Parameters

\begin{tabular}{|c|c|c|c|c|c|}
\hline $\begin{array}{l}\text { Band } \\
\text { (1) }\end{array}$ & $\begin{array}{l}\text { Survey } \\
\text { (2) }\end{array}$ & $\begin{array}{c}\text { Sky Noise } \\
\text { (3) }\end{array}$ & $\begin{array}{c}\text { Sky Level } \\
\text { (4) }\end{array}$ & $\begin{array}{l}\sigma_{\text {Gaussian }} \\
(5)\end{array}$ & $\begin{array}{l}w_{\text {Gaussian }} \\
(6)\end{array}$ \\
\hline \multicolumn{6}{|c|}{ Near Infrared } \\
\hline$[4.5]$ & SERVS & 0.003 & 0.299 & {$[1.0,1.95]$} & {$[0.3,0.7]$} \\
\hline$[3.6]$ & SERVS & 0.002 & 0.099 & {$[1.08,2.20]$} & {$[0.37,0.63]$} \\
\hline$K_{\mathrm{s}}$ & VIDEO & 3.114 & -0.306 & {$[1.60,3.09,10.0]$} & {$[0.59,0.27,0.135]$} \\
\hline$H$ & VIDEO & 2.170 & -0.226 & {$[1.60,3.09,8.64]$} & {$[0.61,0.24,0.15]$} \\
\hline$J$ & VIDEO & 1.418 & -0.169 & {$[1.59,2.94,7.24]$} & {$[0.63,0.30,0.16]$} \\
\hline$Y$ & VIDEO & 1.166 & -0.147 & {$[1.61,3.15,6.51]$} & {$[0.54,0.19,0.27]$} \\
\hline$Z$ & VIDEO & 0.613 & -0.130 & {$[1.62,2.76,4.87,11.80]$} & {$[0.5,0.1,0.32,0.08]$} \\
\hline \multicolumn{6}{|c|}{ Optical } \\
\hline$z^{\prime}$ & CFHTLS-D1 & 0.774 & -0.117 & {$[1.56,2.72,6.21]$} & {$[0.73,0.05,0.23]$} \\
\hline$i^{\prime}$ & CFHTLS-D1 & 0.330 & -0.096 & {$[1.27,2.13,4.35,10.23]$} & {$[0.37,0.42,0.15,0.06]$} \\
\hline$r^{\prime}$ & CFHTLS-D1 & 0.248 & -0.059 & {$[1.37,2.27,4.71,11.45]$} & {$[0.36,0.44,0.16,0.04]$} \\
\hline$g^{\prime}$ & CFHTLS-D1 & 0.173 & -0.031 & {$[1.57,2.71,6.17]$} & {$[0.43,0.44,0.14]$} \\
\hline$u^{\prime}$ & CFHTLS-D1 & 0.258 & -0.003 & {$[1.66,2.84,6.49]$} & {$[0.45,0.42,0.13]$} \\
\hline
\end{tabular}

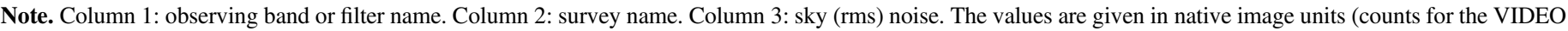

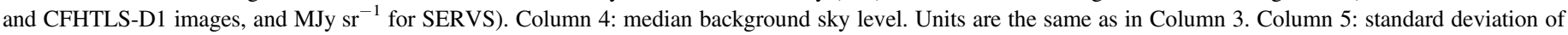

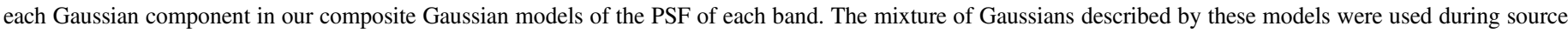

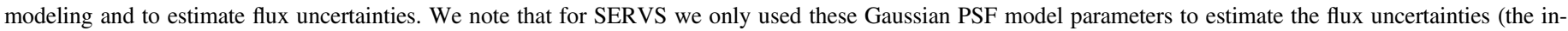

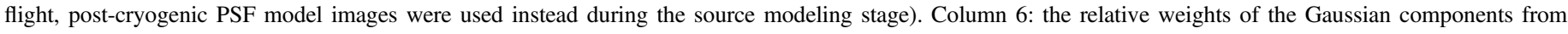
Column 5, normalized to sum to 1.0 .

profile with $n=4$ ) and once using an exponential profile (equivalent to a Sérsic profile with $n=1$ ). The resolved profile fit resulting in the lowest reduced chi squared $\left(\chi_{\text {red }}^{2}\right)$ value after optimization with The Tractor is reported in our final output catalog.

For the IRAC-selected catalog, we extract an image cutout from each band using MONTAGE and create a model of the source and its neighbors. However, since the sources in the IRAC-selected catalog are typically near the SERVS detection limit, we restrict the source surface brightness profile models to be unresolved point sources.

\subsection{Image Calibration Parameters}

After the source model at the fiducial band has been determined, this model is convolved with the appropriate PSF for each band/instrument. We use a mixture of circular Gaussians with 2-4 components each to model the PSFs for the ground-based VIDEO and CFHTLS-D1 data. For each VIDEO and CFHTLS-D1 band, we select sources that are likely to be stars based on their bright (but unsaturated) fluxes, Gaussianlike radial profiles, and high PSTAR values. We estimate the number of composite Gaussians needed to describe the source as well as the Gaussian $\sigma$ values and relative weights by visual inspection. Finally, we use these estimates as initial guesses to obtain least-squares fits to the multi-component Gaussian PSF parameters for each band using The Tractor. These parameters are listed in Table 2. For the SERVS data, the large wings and strong diffraction spikes of the PSF from these diffractionlimited, space-based data led to us using the in-flight postcryogenic IRAC point response functions (PRFs) described in Hora et al. (2012).

We also specify the sky (rms) noise and the median background sky level for each band. These parameters correct for image contamination from sources such as instrument noise and zodiacal light. The sky noise and sky level values used in each band are listed in Table 2 .

\subsection{Optimization}

Given a source with the information described above, The Tractor performs a least-squares fit to the image data to determine the source brightnesses. While in principle all parameters may be left free to vary (i.e., source positions, shapes, and fluxes), we found that allowing too many parameters to vary caused some fits to yield unphysical results. To avoid these issues, we held all image and calibration parameters fixed during optimization except for the fluxes, which are left free to vary. This type of photometric fitting strategy is sometimes referred to as "forced photometry.""17 Examples of the original multi-band images, models, and $\chi^{2}$ maps for a blended IRAC source and a non-blended, faint IRAC source for which The Tractor has produced improved multi-band photometry compared to the original input catalog is shown in Figures 3 and 4.

Our implementation of The Tractor required the development of a parallelized PYTHON driver script. Parallelization was performed using the Multiprocessing PYTHON module. A full run of our script for the 117,281 sources with imaging available over 12 bands in our VIDEO-selected input catalog took approximately $16 \mathrm{hr}$ on a cluster node with 16 cores and $64 \mathrm{~GB}$ of memory. Diagnostic images (original sub-image cutout, source-model image, and $\chi^{2}$ array) may be optionally produced, though this significantly increases the run time of our code.

\footnotetext{
17 We note that usage of the term "forced photometry" is not consistent throughout the literature. In some publications, the term refers to the process of smoothing all images to match the lowest resolution band and then performing matched-aperture photometry. Here, our usage of the term follows from Lang et al. (2016b) and describes the process of using prior information from a highresolution band to model the flux at the same position in lower resolution bands.
} 


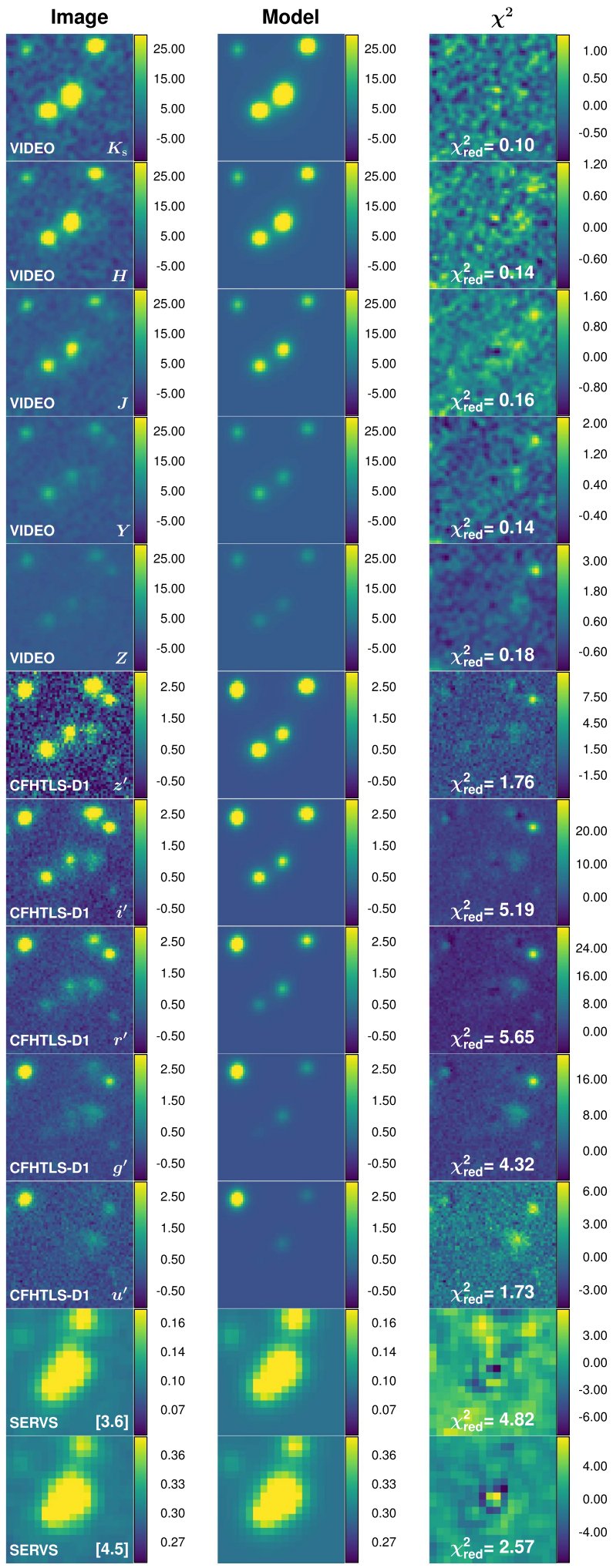

Figure 3. Example of a source that is clearly blended in the SERVS bands but resolved in the fiducial VIDEO band (for this source, $K_{\mathrm{s}}$ band). The cutout dimensions are $10^{\prime \prime} \times 10^{\prime \prime}$ and the source was modeled using a deVaucouleurs profile. The left column shows the original image, the center column shows the source model convolved with the PSF of each band, and the right column shows the $\chi^{2}$ image and $\chi_{\text {red }}^{2}$ value after fitting with The Tractor. The colorbar units are in image counts for VIDEO and CFHTLS-D1 and MJy sr for SERVS.
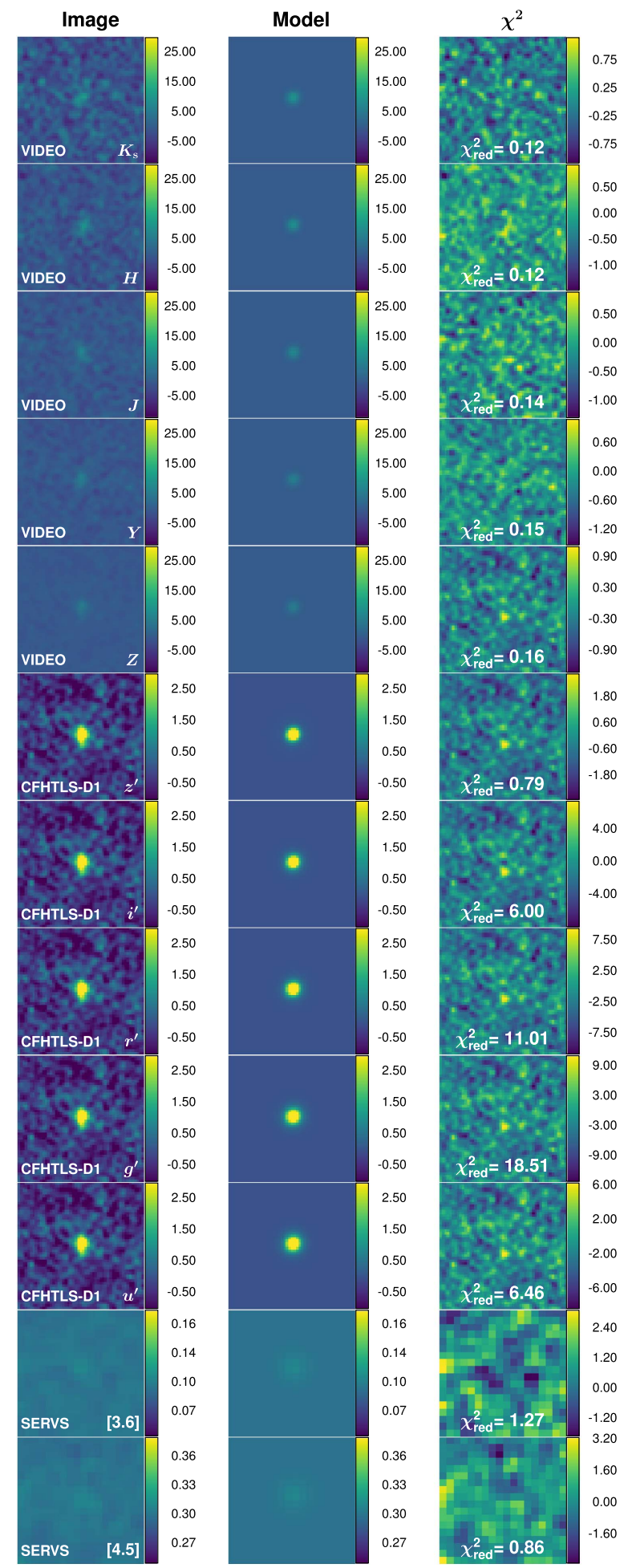

Figure 4. Example of our forced-photometry procedure for a source with no blending issues that is much fainter than the example shown in Figure 3. The cutout dimensions are $10^{\prime \prime} \times 10^{\prime \prime}$ and the source was modeled using a pointsource model. The left column shows the original image, the center column shows the source model convolved with the PSF of each band, and the right column shows the $\chi^{2}$ image and $\chi_{\text {red }}^{2}$ value after fitting with The Tractor. The colorbar units are in image counts for VIDEO and CFHTLS-D1 and MJy sr for SERVS. 


\subsection{Output Catalogs}

The forced photometry of the VIDEO- and IRAC-selected output catalogs produce multi-band measurements of source fluxes and magnitudes as well as errors. Information on the source position, fiducial-band, best-fitting surface brightness model, and $\chi_{\text {red }}^{2}$ value after fitting with The Tractor are also included in the output catalogs. We provide these catalogs in the online supplementary information and present additional details on their contents in the Appendix.

\subsubsection{Saturated Sources}

We note that some of the brightest sources in the VIDEOselected output catalog may be saturated. Thus, we suggest that users wishing to avoid the inclusion of such sources in subsequent analyses utilizing our Tractor VIDEO-selected output catalog consider the binary saturation flag we have provided in the catalog. The saturation flag column identifies sources with a high probability of being saturated based on the comparison between The Tractor and original photometry shown in Figure 5. For the VIDEO data, sources with magnitudes brighter than 14.0 for $K s$ and $H$ band, as well as sources brighter than 14.5, 13.6, and 13.8 for the $J, Y$, and $Z$ bands, respectively, are flagged as saturated. For the CFHTLS-D1 data, sources brighter than 16.3, 15.9, 15.9, 15.1, and 15.7 for the $i^{\prime}, r^{\prime}, g^{\prime}, z^{\prime}$, and $u^{\prime}$ bands, respectively, are flagged. Finally, sources brighter than magnitude 14.0 for the IRAC $3.6 \mu \mathrm{m}$ band and 13.5 for the IRAC $4.5 \mu \mathrm{m}$ band are flagged. For further details, we refer readers to Table 4 in the Appendix.

\subsection{Caveats}

Although our new multi-band photometric catalogs produced using The Tractor offer important advantages over existing catalogs for blended and/or intrinsically faint sources, there are a number of important caveats. We emphasize that improved photometry of blended IRAC sources can only be achieved if the blended objects are well-resolved in the fiducial VIDEO band used to generate the source model. For highly complex, extended sources not well described by a deVaucouleurs or exponential model, the accuracy of our photometry with The Tractor will be reduced. The inclusion of additional surface brightness profile models and/or performing fitting with The Tractor over multiple iterations may help address this issue in the future. We note that our strategy assumes that the source surface brightness profile is the same at all 12 NIR and optical bands included in our analysis. In other words, we effectively assume morphological $k$ corrections are small.

Our photometry also does not take into account spatial variations in the PSF or sky background level, which could lead to aperture errors that are difficult to correct and poorer flux measurement accuracy for fainter sources, respectively. While in principle it would be possible to provide The Tractor with position-dependent PSF information for all bands based on models generated using the PSFEX software (Bertin 2011), this would increase the computational cost substantially.

Although position-dependent astrometric variations can in principle compromise the photometric accuracy of The Tractor, the data sets used here do not suffer significantly from such effects. Both VIDEO and CFHTLS-D1 have relative astrometric uncertainties <0". 1 (Gwyn 2012; Jarvis et al. 2013). For the SERVS data, the IRAC Instrument Handbook ${ }^{18}$ reports that the

\footnotetext{
${ }^{18}$ http://irsa.ipac.caltech.edu/data/SPITZER/docs/irac
}

astrometry is typically accurate to $\sim 0$ ". 2 , or about the size of a single pixel in the VIDEO survey. Given these relatively small uncertainties, we don't expect astrometric errors to be a dominant limiting factor in the accuracy of our forced photometry.

\section{Results}

\subsection{VIDEO-Selected Catalog}

We find that about $65 \%$ of the sources in the VIDEOselected forced-photometry catalog are extended based on the criteria described in Section 3.4 and require spatially resolved surface brightness profile models. The high fraction of extended sources suggests that the number of blended SERVS sources is indeed significantly higher than our lower limit of $17 \%$ (see Section 3.2). Of the resolved sources, the majority are best fit by a deVaucouleurs profile $(\sim 61 \%)$ rather than an exponential profile $(\sim 39 \%)$. The vast majority $(\sim 84 \%)$ of the sources were modeled using the VIDEO $K_{\mathrm{s}}$-band data as the fiducial band.

A comparison of the source magnitudes from The Tractor forced photometry and the original photometry for the VIDEOselected catalog is shown in Figure 5. Our forced photometry is typically in good agreement with the original catalog magnitudes, though some scatter is apparent. As expected, the scatter is largest for faint sources. The photometry of these sources is likely more sensitive to noise fluctuations across the image that have not been accounted for by our constant noise assumption. Other factors that may contribute to the scatter include the presence of blended sources, spatial PSF variations, inaccurately matched sources in the input catalog, and issues with the photometry from the original catalogs. Restricting the comparison to "isolated" sources that lack a neighbor within 3 !' 8 in the VIDEO-selected input catalog and were also fit with point-source models in the Tractor forced photometry further reduces the scatter in Figure 5.

Table 3 summarizes the median difference between our new forced photometry and the original photometry at each band for all sources, blended sources, and isolated point sources (nonblended sources). The magnitudes of the offsets between The Tractor and original catalogs are dominated by sources at or below the original detection thresholds of the respective surveys, a regime in which accurate source brightness measurement is difficult. The uncertainty in the brightnesses of these faint sources is indicated by their large errors in our output multi-band catalog as well as in the original VIDEO, CFHTLS-D1, and SERVS catalogs.

\subsection{IRAC-selected Catalog}

In Figure 6, we compare the SERVS photometry from the IRAC-selected input catalog with the results of our new forced photometry. The photometry in each of these catalogs is generally in good agreement, except for a small population of very faint sources near the SERVS detection limit where the scatter increases notably. These sources may be extended and characterized by inherently low surface brightness emission, making our assumption of a point-source surface brightness profile inadequate. It is also possible that the original photometry overestimated the source brightness for many of these objects by erroneously including noise and/or emission from nearby confusing sources.

Since the IRAC bands themselves were used as priors, no de-blending was possible. The primary benefit of performing forced photometry on the IRAC-selected catalog is the ability 

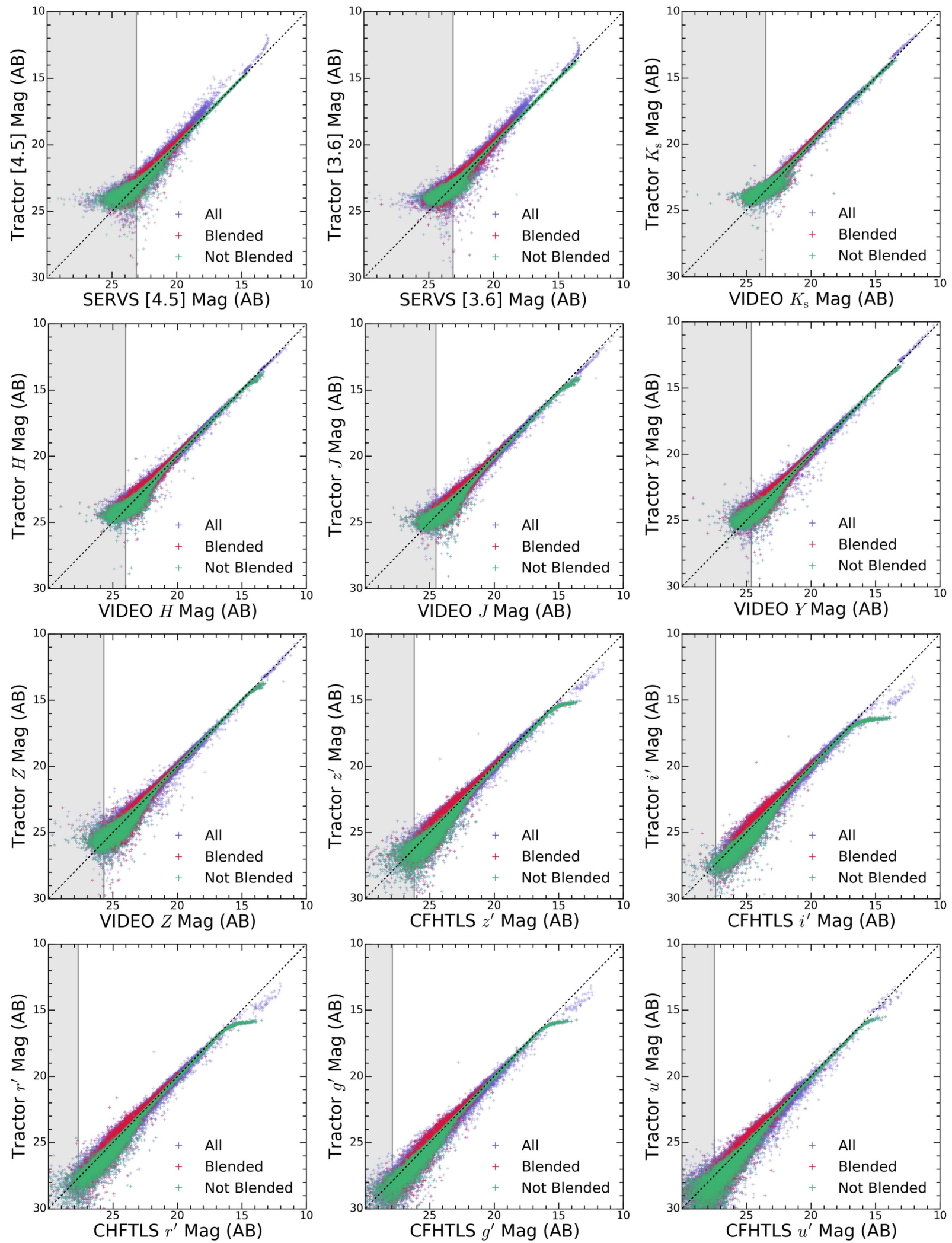

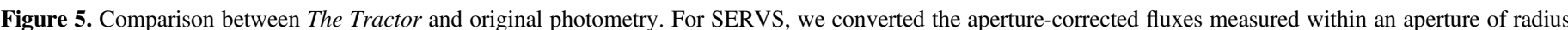

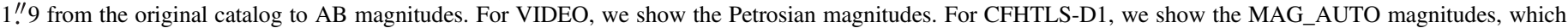

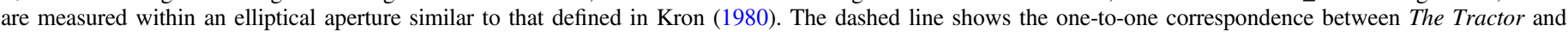

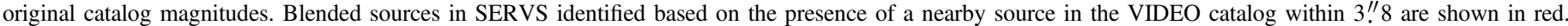

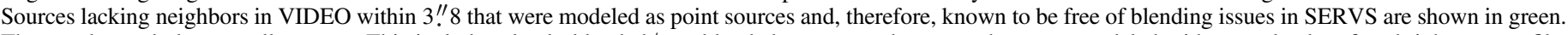

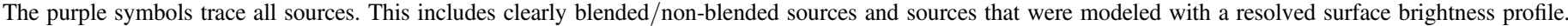
The gray-shaded region highlights the parameter space below the average $5 \sigma$ detection threshold of each survey. 
Table 3

Median Photometric Offsets

\begin{tabular}{|c|c|c|c|c|c|c|}
\hline $\begin{array}{l}\text { Band } \\
\text { (1) }\end{array}$ & $\begin{array}{l}N_{\text {All }} \\
(2)\end{array}$ & $\begin{array}{l}\Delta M_{\mathrm{All}} \\
(3)\end{array}$ & $\begin{array}{l}N_{\text {Blended }} \\
\quad(4)\end{array}$ & $\begin{array}{c}\Delta M_{\text {Blended }} \\
\quad(5)\end{array}$ & $\begin{array}{l}N_{\text {Not Blended }} \\
\quad \text { (6) }\end{array}$ & $\Delta M_{\text {Not }}$ (7) \\
\hline \multicolumn{7}{|c|}{ Near Infrared } \\
\hline $3.6 \mu \mathrm{m}$ & 103911 & -0.235 & 15808 & -0.175 & 28657 & -0.097 \\
\hline$K_{\mathrm{s}}$ & 98811 & -0.230 & 15928 & -0.215 & 23755 & -0.111 \\
\hline$H$ & 104752 & -0.176 & 17129 & -0.154 & 25598 & -0.035 \\
\hline$Z$ & 107651 & -0.063 & 17714 & -0.013 & 28601 & 0.040 \\
\hline \multicolumn{7}{|l|}{ Optical } \\
\hline$\overline{z^{\prime}}$ & 104891 & -0.138 & 17286 & -0.008 & 29651 & 0.233 \\
\hline$i^{\prime}$ & 105392 & -0.008 & 17332 & -0.009 & 29841 & 0.224 \\
\hline$r^{\prime}$ & 105044 & 0.006 & 17256 & 0.004 & 29714 & 0.198 \\
\hline
\end{tabular}

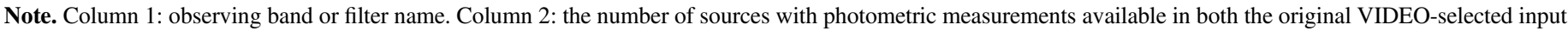

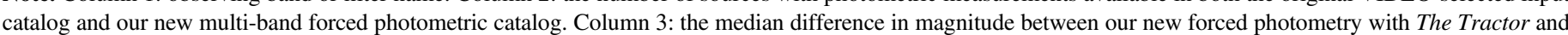

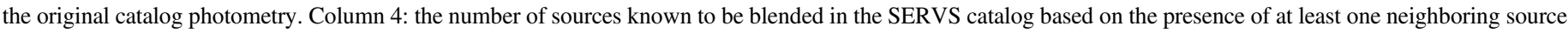

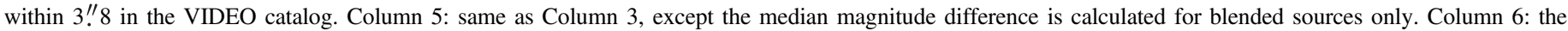

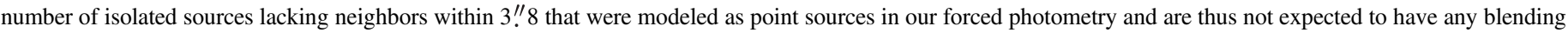

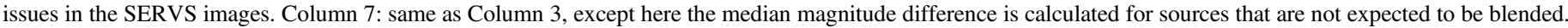

to identify faint VIDEO and CFHTLS-D1 counterparts to extremely red sources only detected previously in the IRAC bands. Of the 8441 sources in the IRAC-selected input catalog, ${ }^{19}$ photometric measurements at $K_{\mathrm{s}}$-band were possible using The Tractor for $\approx 69 \%$ of the sample. We emphasize that this population of new $K_{\mathrm{s}}$-band detections represents intrinsically faint sources that fall below the detection threshold in the VIDEO single-band catalogs, but can be successfully measured with our forced-photometry approach.

Figure 7 compares the number distribution of $K_{\mathrm{s}}$-band source magnitudes from the original VIDEO-selected input catalog with measurements from our new IRAC-selected forced photometric catalog. The distribution of new $K_{\mathrm{s}}$-band source magnitudes clearly demonstrates that our forced photometry detects a population of extremely faint, red objects that fall below the single-band detection threshold in the original VIDEO photometry. Such intrinsically faint sources at or slightly below the original image detection threshold can only be detected with statistical techniques such as forced photometry that incorporate prior information about the source position from a detection at another band.

\subsection{Depth}

In Figures 8 and 9, we show the magnitude $\operatorname{error}^{20}$ as a function of magnitude for each band of the VIDEO- and IRACselected Tractor catalogs. We use these figures to determine the $5 \sigma$ survey depth for each band by measuring the location in the distribution of magnitudes where the faintest sources reach a magnitude error of 0.2. For the two IRAC bands at 3.6 and $4.5 \mu \mathrm{m}$, we find $5 \sigma$ limits of 23.26 and 23.59 in the VIDEO-

\footnotetext{
$\overline{19}$ We refer readers to Section 3.2 for details on the construction of the IRACselected input catalog.

${ }^{20}$ For a detailed discussion of the calculation of flux and magnitude errors, we refer readers to the Appendix.
}

selected output catalog, and $5 \sigma$ limits of 23.26 and 22.86 in the IRAC-selected catalog. Values of the $5 \sigma$ depths for the remaining bands are provided in Figures 8 and 9. The $5 \sigma$ depths for our output forced-photometry catalogs are generally comparable to the magnitude limits from the original catalogs shown in Table 1 or slightly deeper.

\section{Discussion}

\subsection{Colors}

In Figure 10, we show the $3.6 \mu \mathrm{m}$ versus $J-K_{\mathrm{S}}$ colors for the original VIDEO-selected input catalog (top left panel) and our new forced photometry (top right panel). The same sources are shown in the top left and right panels-the only difference is the photometric catalog used to compute the colors. Compared to the top left panel of Figure 10, the top right panel showing the photometry from The Tractor has less scatter in the distribution of Lyman break galaxy (LBG)selected sources $\left(g^{\prime}-r^{\prime}<1.2\right.$ and $u^{\prime}-g^{\prime}>g^{\prime}-r^{\prime}+1$; Steidel et al. 2002) residing in the redshift range $2.7<z<3.3$. Figure 10 also clearly indicates that the stellar locus at $J-K_{\mathrm{s}} \approx-0.2$ is substantially better defined when the source colors are computed using The Tractor photometry. This qualitatively suggests that the colors, and therefore the underlying photometric measurements, are more robust in our forced-photometry catalog compared to the original input catalog. We provide a more quantitative assessment of the improved photometric accuracy of our forced-photometry catalog in Section 5.2.

In the bottom panel of Figure 10, we show the NIR colors based on our forced photometry as in the middle panel, but this time we also highlight sources that were not detected in the original catalog. This population of sources that are only identified in our new VIDEO-selected forced photometric catalog has a large degree of scatter, but this is expected given 
Table 4

Table Columns and their Descriptions for the VIDEO- and IRAC-selected Output Tractor Catalogs

\begin{tabular}{|c|c|c|c|}
\hline Name & Description & Name & Description \\
\hline R.A. & J2000 position from original VIDEO catalog & $3.6 \mathrm{mag}$ & SERVS [3.6] magnitude \\
\hline Decl. & J2000 position from original VIDEO catalog & $4.5 \mathrm{mag}$ & SERVS [4.5] magnitude \\
\hline Flux-Ks & VIDEO $K s$-band flux & e_Ks mag & VIDEO $K s$-band magnitude error \\
\hline Flux- $H$ & VIDEO $H$-band flux & e_H mag & VIDEO $H$-band magnitude error \\
\hline Flux- $J$ & VIDEO $J$-band flux & e_J mag & VIDEO $J$-band magnitude error \\
\hline Flux- $Y$ & VIDEO $Y$-band flux & e_Y mag & VIDEO $Y$-band magnitude error \\
\hline Flux- $Z$ & VIDEO Z-band flux & e_Z mag & VIDEO Z-band magnitude error \\
\hline Flux- $i^{\prime}$ & CFHTLS-D $1 i^{\prime}$-band flux & $\mathrm{e} \_i^{\prime} \mathrm{mag}$ & CFHTLS-D1 $i^{\prime}$-band magnitude error \\
\hline Flux- $r^{\prime}$ & CFHTLS-D1 $r^{\prime}$-band flux & $\mathrm{e} \_r^{\prime} \mathrm{mag}$ & CFHTLS-D1 $r^{\prime}$-band magnitude error \\
\hline Flux- $g^{\prime}$ & CFHTLS-D1 $g^{\prime}$-band flux & $\mathrm{e} \_g^{\prime} \mathrm{mag}$ & CFHTLS-D1 $g^{\prime}$-band magnitude error \\
\hline Flux $-z^{\prime} \mathrm{C}$ & CFHTLS-D1 $z^{\prime}$-band flux & $\mathrm{e} \_z^{\prime} \mathrm{Cmag}$ & CFHTLS-D1 $z^{\prime}$-band magnitude error \\
\hline Flux- $u^{\prime}$ & CFHTLS-D1 $u^{\prime}$-band flux & $\mathrm{e} \_u^{\prime} \mathrm{mag}$ & CFHTLS-D1 $u^{\prime}$-band magnitude error \\
\hline Flux-3.6 & SERVS [3.6] flux & e_3.6 mag & SERVS [3.6] magnitude error \\
\hline Flux-4.5 & SERVS [4.5] flux & e_4.5 mag & SERVS [4.5] magnitude error \\
\hline e_Flux-Ks & VIDEO $K s$-band flux error & redchi-Ks & VIDEO $K s$-band $\chi_{\text {red }}^{2}$ \\
\hline e_Flux-H & VIDEO $H$-band flux error & redchi-H & VIDEO $H$-band $\chi_{\text {red }}^{2}$ \\
\hline e_Flux-J & VIDEO $J$-band flux error & redchi-J & VIDEO $J$-band $\chi_{\text {red }}^{2}$ \\
\hline e_Flux-Y & VIDEO $Y$-band flux error & redchi-Y & VIDEO $Y$-band $\chi_{\text {red }}^{2}$ \\
\hline e_Flux-Z & VIDEO Z-band flux error & redchi-Z & VIDEO Z-band $\chi_{\text {red }}^{2}$ \\
\hline e_Flux- $i^{\prime}$ & CFHTLS-D1 $i^{\prime}$-band flux error & redchi- $i^{\prime}$ & CFHTLS-D1 $i^{\prime}$-band $\chi_{\text {red }}^{2}$ \\
\hline e_Flux- $r^{\prime}$ & CFHTLS-D1 $r^{\prime}$-band flux error & redchi- $-r^{\prime}$ & CFHTLS-D $1 r^{\prime}$-band $\chi_{\text {red }}^{2}$ \\
\hline e_Flux- $g^{\prime}$ & CFHTLS-D1 $g^{\prime}$-band flux error & redchi- $g^{\prime}$ & CFHTLS-D1 $g^{\prime}$-band $\chi_{\text {red }}^{2}$ \\
\hline e_Flux $-z^{\prime} \mathrm{C}$ & CFHTLS-D1 $z^{\prime}$-band flux error & redchi- $z^{\prime} \mathrm{C}$ & CFHTLS-D1 $z^{\prime}$-band $\chi_{\text {red }}^{2}$ \\
\hline e_Flux- $u^{\prime}$ & CFHTLS-D1 $u^{\prime}$-band flux error & redchi- $u^{\prime}$ & CFHTLS-D $1 u^{\prime}$-band $\chi_{\text {red }}^{2}$ \\
\hline e_Flux-3.6 & SERVS [3.6] flux error & redchi-3.6 & SERVS [3.6] $\chi_{\text {red }}^{2}$ \\
\hline e_Flux-4.5 & SERVS [4.5] flux error & redchi-4.5 & SERVS [4.5] $\chi_{\text {red }}^{2}$ \\
\hline Ks mag & VIDEO $K s$-band magnitude & FiducialBand & VIDEO band used to define source model \\
\hline H mag & VIDEO $H$-band magnitude & SourceModel & Source surface brightness profile model \\
\hline $\mathrm{J}$ mag & VIDEO $J$-band magnitude & Sat-flag & Source saturation flag in each band \\
\hline Y mag & VIDEO $Y$-band magnitude & $\cdots$ & $\cdots$ \\
\hline $\mathrm{Z}$ mag & VIDEO Z-band magnitude & $\cdots$ & $\cdots$ \\
\hline$i^{\prime} \mathrm{mag}$ & CFHTLS-D1 $i^{\prime}$-band magnitude & $\ldots$ & $\ldots$ \\
\hline$r^{\prime}$ mag & CFHTLS-D1 $r^{\prime}$-band magnitude & $\cdots$ & $\cdots$ \\
\hline$g^{\prime} \mathrm{mag}$ & CFHTLS-D1 $g^{\prime}$-band magnitude & $\ldots$ & $\ldots$ \\
\hline$z^{\prime} \mathrm{C}$ mag & CFHTLS-D1 $z^{\prime}$-band magnitude & $\cdots$ & $\ldots$ \\
\hline$u^{\prime}$ mag & CFHTLS-D1 $u^{\prime}$-band magnitude & $\cdots$ & $\cdots$ \\
\hline
\end{tabular}

Note. All fluxes are given in units of $\mu \mathrm{Jy}$ and all magnitudes are based on the AB system. A description of the calculation of flux and magnitude uncertainties is given in the Appendix. SourceModel values may be one of the following: Dev (deVaucouleurs profile), Exp (exponential profile), or PtSrc (point-source model). For the Satflag column, values are a single binary string containing a 0 (not saturated) or a 1 (saturated) for each band in the following order: $K s, H, J, Y, Z, i^{\prime}, r^{\prime}, g^{\prime}, z^{\prime}, u^{\prime}, 3.6 \mu \mathrm{m}$, and $4.5 \mu \mathrm{m}$. The Sat-flag column is only included for the VIDEO-selected output source catalog since saturation is not an issue in the IRAC-selected catalog of intrinsically fainter sources.

(This table is available in its entirety in machine-readable form.)

the intrinsically faint nature of many of these objects. We emphasize that some of these sources do in fact lie within the main locus of galaxy colors, but were simply too faint to be detected in the original VIDEO photometry. Thus, for studies geared toward intrinsically faint and potentially rare source populations, our implementation of photometry with The Tractor offers improved sensitivity compared to traditional positional matching methods.

\subsection{Photometric Redshifts}

\subsubsection{Distribution}

One of the primary motivations for improving the accuracy of the original multi-band photometry is to obtain more robust photometric redshifts. To test whether we have accomplished this in our test field, we derived photometric redshifts based on the 12 NIR and optical SERVS, VIDEO, and CFHTLS-D1 data described in this study using HyperZ (Bolzonella et al. 2000). Our galaxy SED template set up follows that of Pforr et al. (2013), which is based on stellar population models from Maraston (2005). A detailed description of our application of SED fitting and subsequent determination of photometric redshifts will be presented in J. Pforr et al. (2017, in preparation).

In Figure 11, we show the number distribution of photometric redshifts based on the original position-matched source catalogs and the new catalogs constructed using The Tractor. For each catalog, only sources with accurate $\left(\chi_{\text {red }}^{2} \leqslant 3.0\right)$ photometric redshifts and measurements in all 12 bands are shown. Both distributions are clearly dominated by lower-redshift sources, in 

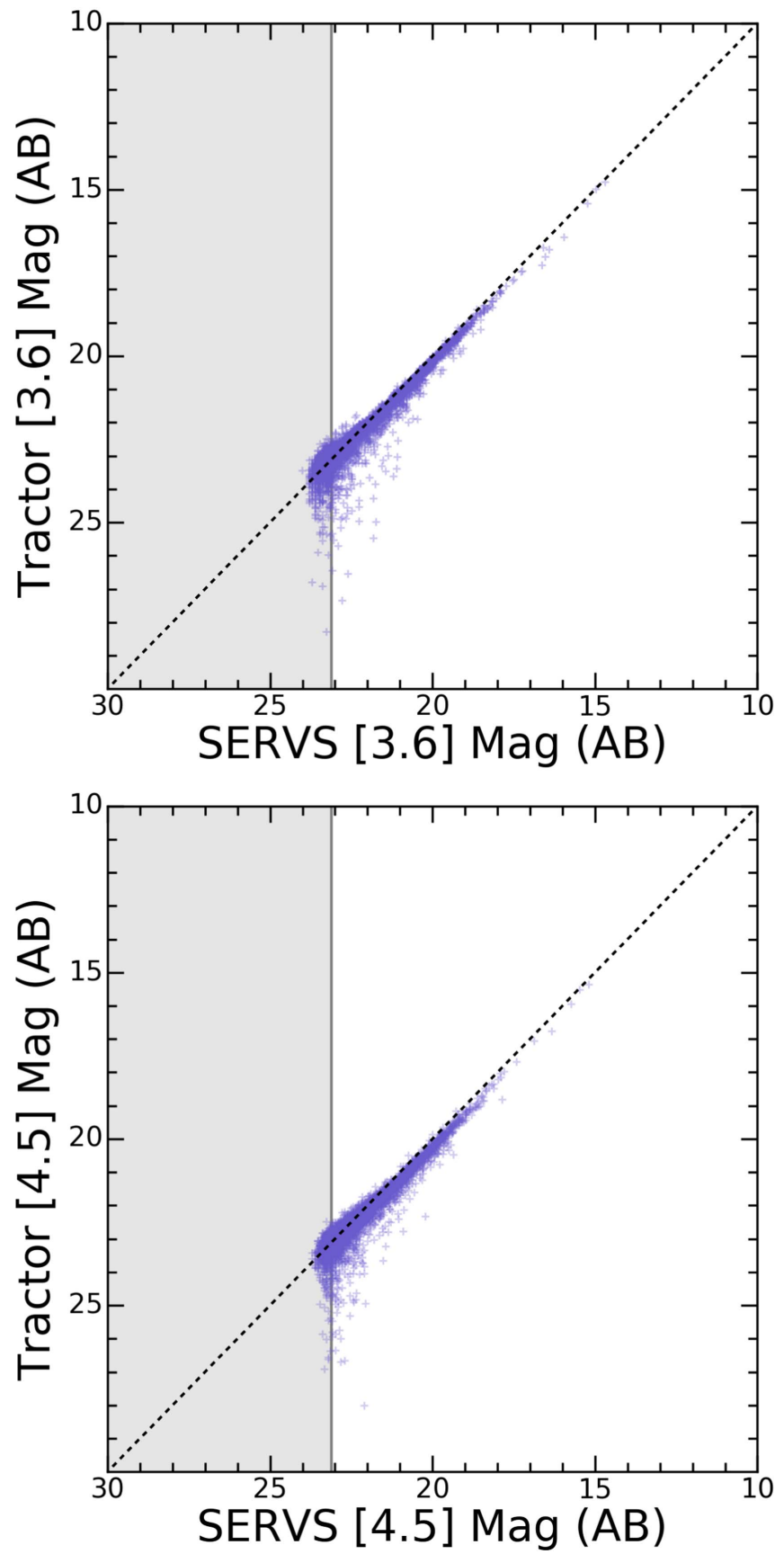

Figure 6. Comparison of our new forced photometry and the IRAC-selected input catalog for the [3.6] (top) and [4.5] (bottom) SERVS bands. We use the original SERVS single-band aperture-corrected catalog photometry measured within an aperture of radius $1 . ! 9$ and converted to AB magnitudes. The grayshaded region highlights the parameter space below the average $5 \sigma$ detection threshold of the SERVS data. The $x$ - and $y$-axis data ranges match those from the VIDEO-selected catalog comparison plots shown in Figure 5.

harmony with the high proportion of sources modeled with resolved surface brightness profiles described in Section 4.1 that are typically associated with lower-redshift objects. We note that the predominance of lower-redshift sources is not unexpected given that this redshift range covers the largest volume of our survey. Due to our sensitivity limitations, we only detect the most luminous galaxies in the highest redshift bins.

The comparison between the original and forced-photometry-based photometric redshifts shown in this figure is striking. When using the forced-photometry source catalog, we obtain accurate photometric redshifts that incorporate all 12 bands into the SED fitting for over twice as many sources compared to the original position-matched photometry $(52,166$ versus 24,273). Furthermore, the number of high-redshift $(z>4.0)$ photometric redshifts sharply increases as well when the forced photometry is used. Based on the original catalog, only 9 high-redshift sources are identified, though none of these are beyond $z=5$. In contrast, we find 70 candidate highredshift sources when using our new forced photometry as input to HyperZ, 5 of which lie in the range $5<z<6$. Thus, a clear advantage of using The Tractor is a substantial increase in the number of sources with robust photometric redshifts and improved sensitivity to faint, potentially high-redshift sources.

\subsubsection{Spectroscopic Redshift Comparison}

Photometric redshifts from HyperZ based on the original, multi-band, position-matched catalogs and our new forced photometry are compared to high-quality spectroscopic redshifts $^{21}$ from the VIMOS VLT Deep Survey (VVDS; Le Fèvre et al. 2013) and the VIMOS Ultra-Deep Survey (VUDS; Le Fèvre et al. 2015) in Figure 12. The top left and right panels of this figure show photometric redshifts from the original VIDEO-selected input catalog and our new forced photometry, respectively. As expected given the known prevalence of VIDEO sources that are blended in the SERVS photometry, Figure 12 illustrates that the photometric and spectroscopic redshifts are much more tightly correlated when the photometric redshifts are determined using forced photometry. Figure 12 also shows the standard deviation $(\sigma)$ of the normalized residuals between the spectroscopic and photometric redshifts $\left(\Delta z_{\text {norm }}\right)$ and the outlier fraction $\left(f_{\text {outlier }}\right)$ for each photometric catalog. These quantities are defined in Equations (1) and (2),

$$
\begin{gathered}
\Delta z_{\text {norm }}=\frac{z_{\text {spec }}-z_{\text {phot }}}{1+z_{\text {spec }}} \\
f_{\text {outlier }}=\left|\Delta z_{\text {norm }}\right|>0.15 .
\end{gathered}
$$

For the 1728 sources with accurate photometric redshifts $\left(\chi_{\text {red }}^{2} \leqslant 3.0\right)$, the original and forced-photometry catalogs have $\sigma=0.23$ and $\sigma=0.08$, respectively. This reduction in scatter for the forced photometry is consistent with the improvement in the $f_{\text {outlier }}$ value, which is $6.54 \%$ in the original photometry and $1.50 \%$ in our new photometric catalog based on The Tractor. To quantify the reduction in $\Delta z_{\text {norm }}$ for the forced photometric catalog, we perform a two-sample Kolmogorov-Smirnov test (Feigelson \& Babu 2012) on $\Delta z_{\text {norm }}$ from the original and Tractor catalogs. This test yields a probability of $p=5.6 \times 10^{-5}$ that the two samples are drawn from the same parent distribution, verifying that the reduction in the scatter for the normalized redshift residuals using forced photometry is indeed statistically significant. This remarkable improvement is largely driven by the fact that The Tractor photometry provides photometric measurements for a larger number of bands included in our study compared to the original photometry based on the positionmatched catalogs.

\footnotetext{
21 The VVDS and VUDS magnitude-limited redshift surveys are based on multi-slit spectroscopy over the wavelength range $3600 \lesssim \lambda \lesssim 9350 \AA$ and include galaxies up to redshift $z \sim 6.7$.
} 

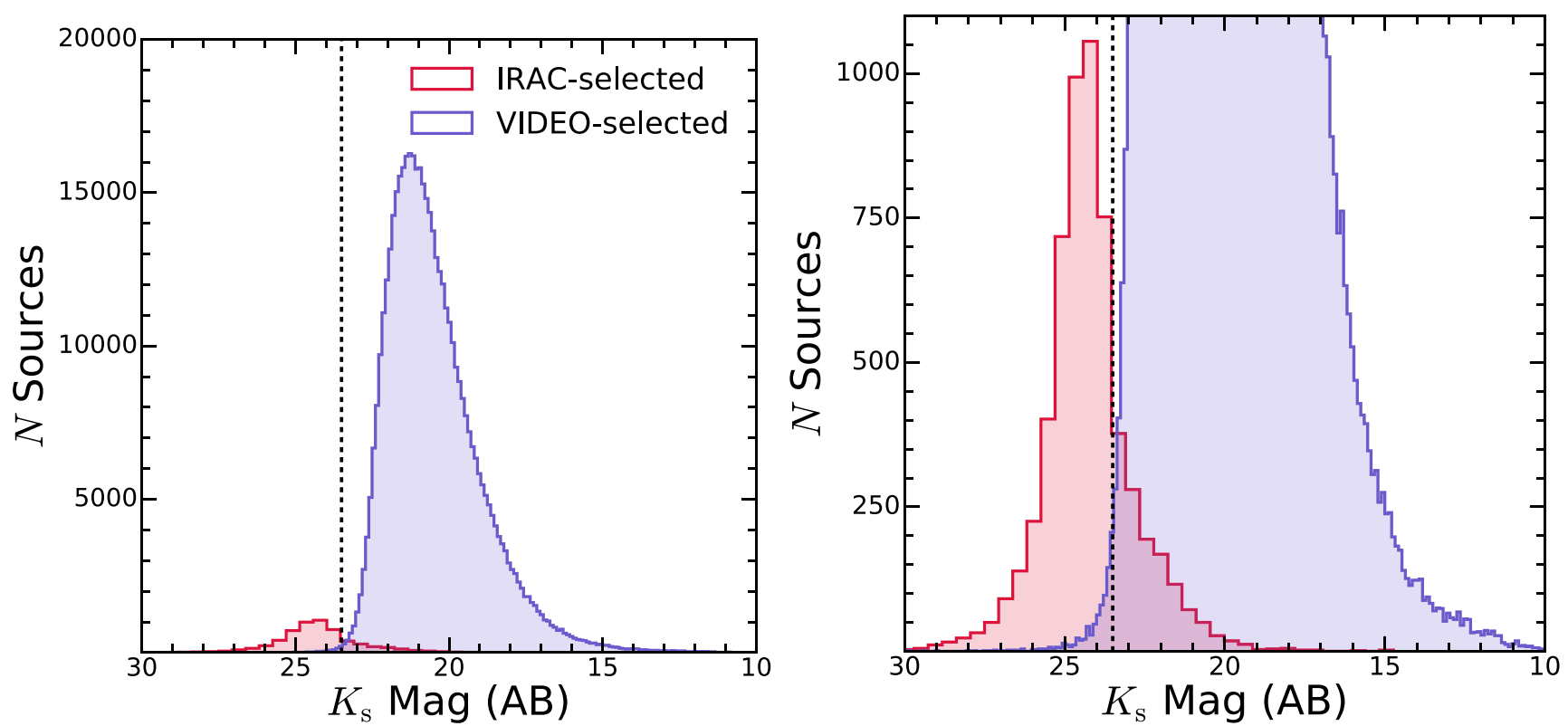

Figure 7. Left: comparison of the number distribution of $K_{\mathrm{s}}$-band source magnitudes from the original VIDEO catalog (purple) and our forced-photometry measurements based on the IRAC-selected input catalog (red). The VIDEO-selected histogram represents Petrosian source magnitudes. We emphasize that sources with measurements based on our IRAC-selected forced photometry were not detected in the original VIDEO catalog. Right: zoomed-in view of the left panel that highlights the distribution of $K_{\mathrm{s}}$-band source magnitudes from our new IRAC-selected forced photometry. The vertical dashed line marks the original VIDEO $K_{\mathrm{s}}$-band $5 \sigma$ detection threshold from Table 1 .

\subsection{Future Science Applications}

The multi-band forced photometry of the VIDEO-selected input catalog provides a number of improvements over the original position-matched catalog in key areas including source matching accuracy, IRAC source de-blending, and sensitivity to faint sources below the single-band detection threshold in a given survey. We have demonstrated that these improvements to the photometry lead to more accurate photometric redshifts, and in the future we plan to use our new forced photometric catalog to accurately measure galaxy masses to study stellar mass assembly out to $z \sim 5$. We will also identify quasar candidates over a wide range of redshifts based on their NIR/optical colors (e.g., following an analysis similar to Richards et al. 2015), taking advantage of our accurate photometry to study the demographics of obscured/unobscured quasars in different cosmic epochs. This will allow us to assess the importance of AGN feedback and how it has evolved over the last 12 billion years.

The forced photometry of the IRAC-selected input catalog, which contains sources with IRAC detections in the original SERVS photometry but no counterparts in any of the original VIDEO source catalogs, showed substantial improvement in the number of source detections in the VIDEO and CFHTLS-D1 bands. At the $K_{\mathrm{s}}$-band alone, the source detection fraction increased dramatically from $0 \%$ in the original catalogs to $69 \%$ after performing forced photometry with The Tractor. This has important implications for the study of extremely red objects (EROs) that are detected in one or more of the SERVS bands but are not detected in any of the original VIDEO photometry. EROs are believed to be extremely dust-enshrouded, high-redshift galaxies with high star formation rates, and represent an evolutionary stage of rapid assembly (e.g., Yan et al. 2004; Wang et al. 2012). Despite the relevance of these objects to our understanding of galaxy formation and growth, large samples of EROs are currently lacking. A future analysis of the SEDs and photometric redshifts of EROs identified in SERVS and analyzed with our implementation of The Tractor will provide much needed information on the properties and demographics of these objects, and address important galaxy evolution questions such as the fraction of obscured star formation missed by optical surveys.

We plan to expand our forced-photometry implementation to the entire XMM-LSS field as well as the remaining four SERVS fields. Given the availability of comparatively deep ground-based NIR and optical data, this will lead to accurate photometric redshift measurements over a large sky footprint, allowing us to maximize the scientific return of the SERVS project. In the NIR, new data from the VISTA Extragalactic Infrared Legacy Survey (Hönig et al. 2017) will provide additional deep data in the $J$ and $K_{\mathrm{S}}$ bands.

Deep optical imaging have recently been made publicly available by the Hyper Suprime-Cam Subaru Strategic Program Data Release 1 (Aihara et al. 2017), which will allow us to perform forced photometry on the full XMM-LSS field along with the EN1 field later in 2017. The first data release of the Panoramic Survey Telescope and Rapid Response System (Pan-STARRS) catalog of optical imaging over five bands covering $3 \pi$ steradian was recently made publicly available as well (Flewelling et al. 2016). However, to obtain comparable photometric redshift accuracy to that presented in this work for one square degree of the XMM-LSS field, we will require optical imaging of comparable depth to that of CFHTLS-D1 from the Pan-STARRS Medium Deep Survey (Huber \& PS1-IPP Team 2015). This survey covers four of the five SERVS fields (XMM-LSS, EN1, LH, and CDFS) and is expected to be released later in the year. Deep optical imaging over the full ES1 and CDFS fields has recently been made available as part of the VST Optical Imaging of the CDFS and ES1 Fields (VOICE; Vaccari et al. 2017), and additional optical imaging of these fields (along with the XMM-LSS field) from the full-depth data release of the Dark Energy Survey (DES; Dark Energy Survey Collaboration et al. 2016) is expected to be made publicly available in 2020 . 

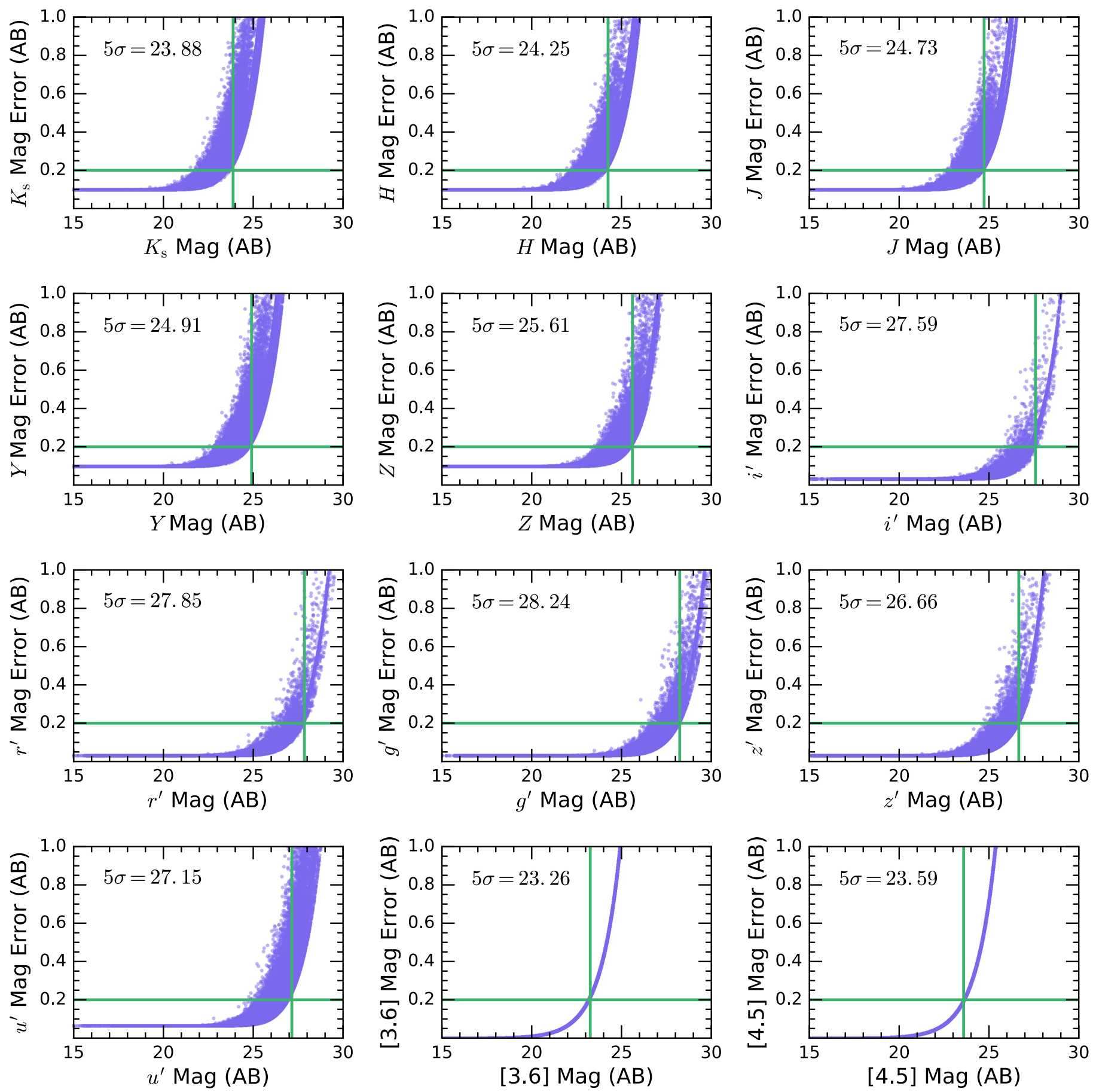

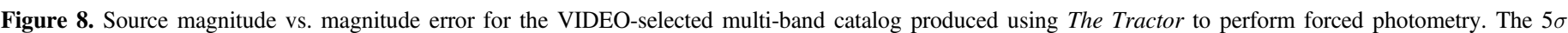

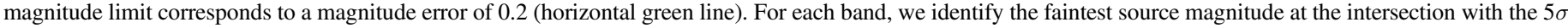
limit (vertical green line). We provide the value of the $5 \sigma$ detection threshold for each band in the upper left corner of each plot.

We will also perform forced photometry on images from the Spitzer DEEPDRILL survey (P.I. Mark Lacy), which will provide post-cryogenic IRAC imaging to $\mu \mathrm{Jy}$ depth of the four predefined Deep Drilling Fields for the Large Synoptic Survey Telescope distributed over an area of $38.4 \mathrm{deg}^{2}\left(1 \mathrm{Gpc}^{3}\right.$ at $z>2$ ). Science highlights of the DEEPDRILL survey include the detection of all the $>10^{11} M_{\odot}$ galaxies out to $z \sim 6$ and the identification of $\sim 40$ protoclusters at $z>2$, which will provide numerous targets of interest for follow-up with JWST. As is the case for SERVS, the legacy value of DEEPDRILL directly hinges upon the the availability of accurate multi-band photometry. Thus, our application of forced photometry with The Tractor presented here will serve as an essential tool for ensuring the scientific success of both SERVS and DEEPDRILL and will provide many important new insights into the physics of galaxy formation and evolution.

\section{Summary}

We have provided a description of our parallelized implementation of The Tractor to perform forced photometry 

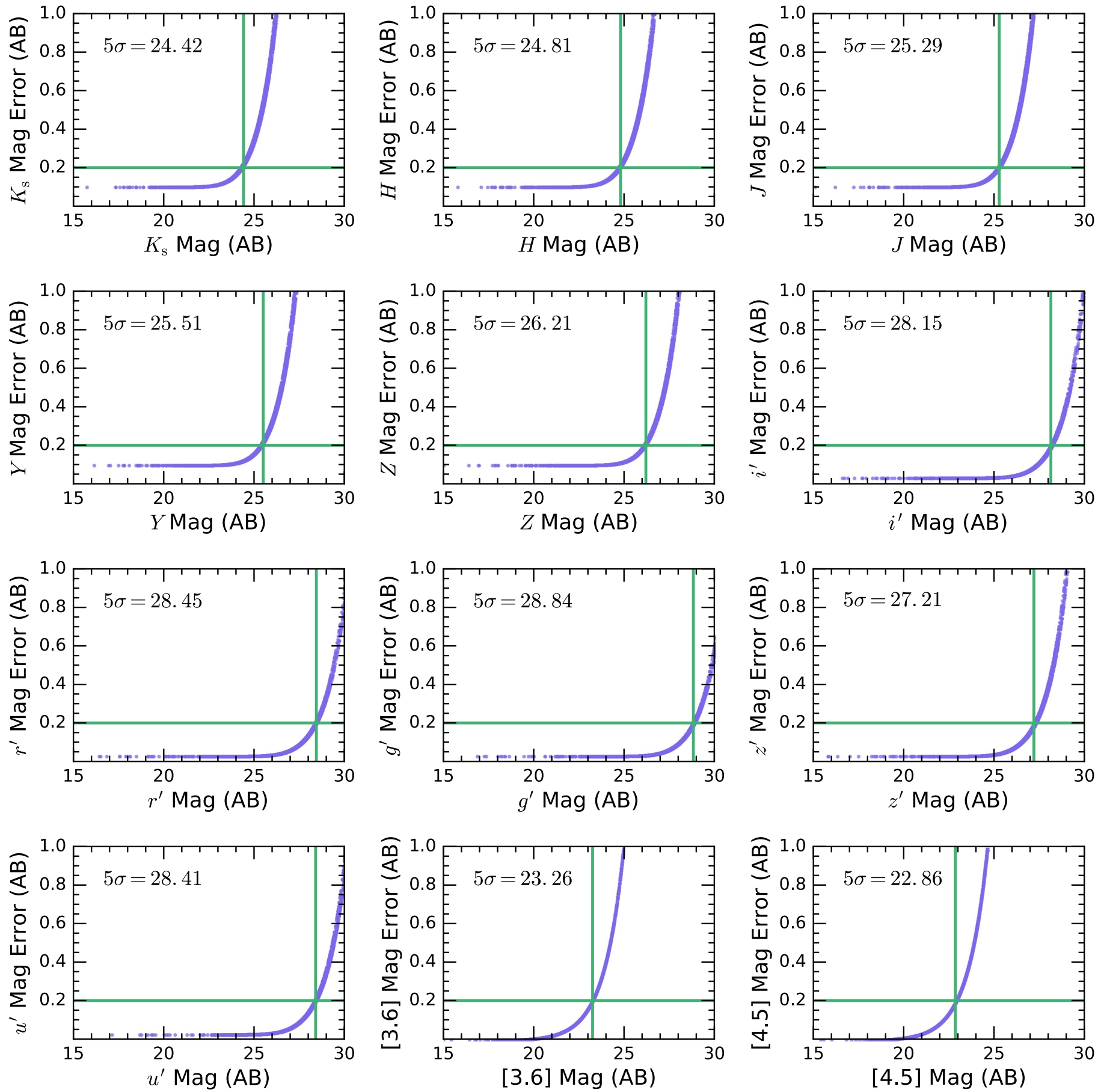

Figure 9. Source magnitude vs. magnitude error for the IRAC-selected multi-band catalog produced using The Tractor to perform forced photometry. The $5 \sigma$ magnitude limit corresponds to a magnitude error of 0.2 (horizontal green line). For each band, we identify the faintest source magnitude at the intersection with the $5 \sigma$ limit (vertical green line). We provide the value of the $5 \sigma$ detection threshold for each band in the upper left corner of each plot.

on 12 NIR and optical bands from SERVS, VIDEO, and CFHTLS-D1 over a square degree of the XMM-LSS field. The VIDEO- and IRAC-selected input catalogs - which have 117,281 and 8441 sources, respectively-are used to define the fiducial source positions that establish the location at which a given source is modeled in each band. For the VIDEOselected input catalog, we found that use of The Tractor lead to the following key advantages compared to position-matched multi-band photometry.
1. By modeling the surface brightness profile of each source in a fiducial, high-resolution VIDEO band and performing forced photometry with The Tractor, we were able to de-blend these objects in the SERVS IRAC images and more accurately measure their photometric properties. This naturally lead to more accurate source cross-identification, as evidenced by the improved definition of the stellar locus for The Tractor photometry shown in the color-color plot comparison in Figure 10. The importance of these 

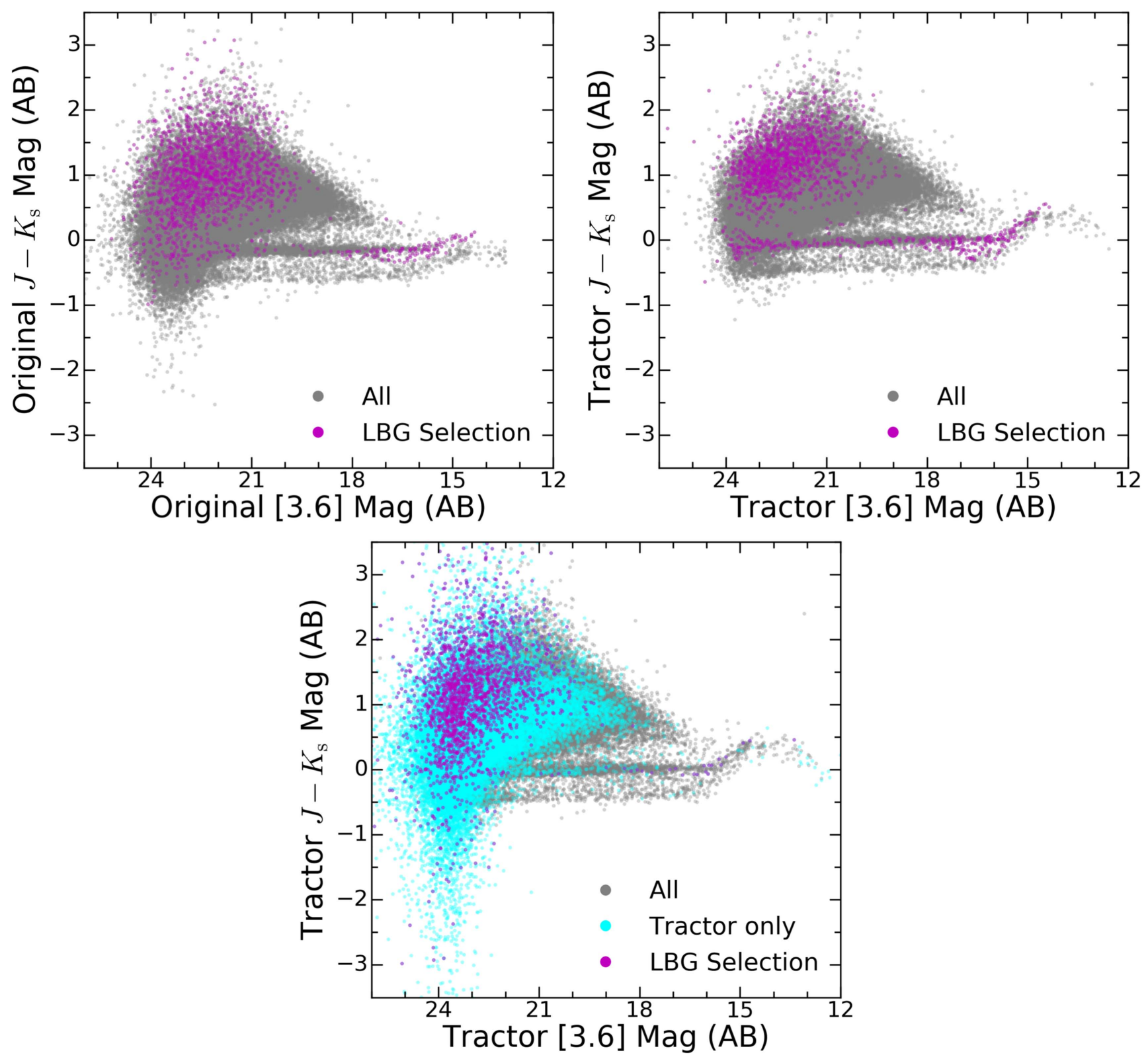

Figure 10. Top left: comparison of the SERVS $3.6 \mu \mathrm{m}$ magnitudes vs. the $J-K_{\mathrm{s}}$ VIDEO colors using the original photometry from the VIDEO-selected input source catalog (Section 3.2). Magnitudes are based on Petrosian, MAG_AUTO, and 1"!9 apertures for VIDEO, CFHTLS-D1, and SERVS, respectively. All sources with detections in the [3.6], $K_{\mathrm{S}}, J, u^{\prime}, g^{\prime}$, and $r^{\prime}$ bands (77,809 sources) are shown as gray symbols. Sources that satisfy the $2.7<z<3.3 \mathrm{LBG}$ criteria of Steidel et al. (2002) are highlighted in magenta. The population of candidate LBG sources that lie on the stellar locus are likely low-redshift interlopers, such as Galactic halo mainsequence stars (e.g., K subdwarfs; Steidel et al. 2003). Top right: same as the top left panel, except here all magnitudes are based on our new forced photometry. Bottom: same as the middle panel, except the additional 30,198 sources that only have measurements in our new forced-photometry catalog (i.e., those that were upper limits in the original input catalog) are shown in cyan.

improvements is highlighted by our estimated lower limit of $17 \%$ for the number of sources that are clearly resolved in the VIDEO images, but blended in the lower-resolution SERVS data.

2. Our application of multi-band forced photometry provided a higher fraction of source detections in each band. This resulted in a factor of two increase in the number of sources with photometric redshift measurements with constraints in all of our optical/NIR bands (Figure 11). As a direct consequence of this, we were able to identify a greater number of candidate high-redshift sources in our square-degree test region. While our new forced-photometry-based photometric redshifts identified 70 objects in the redshift range of $5<z<6$, the position-matched catalogs detected none.

3. Based on comparisons between the photometric redshifts derived from the position-matched and forced-photometry catalogs with spectroscopic redshift measurements from the literature, we found that The Tractor multi-band photometry lead to a statistically significant improvement in photometric redshift accuracy (Figure 12). This will motivate follow-up analyses of various galaxy properties 

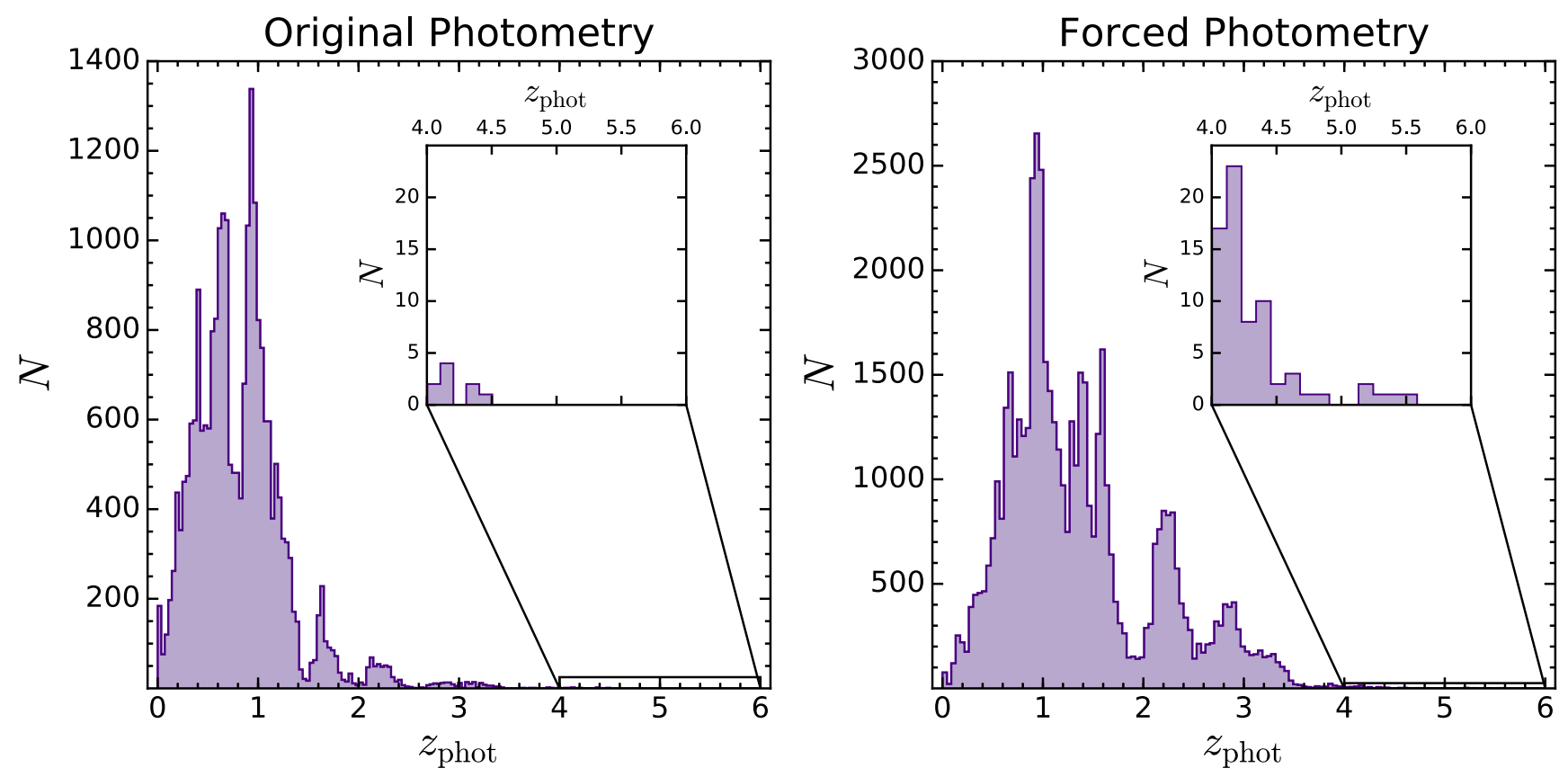

Figure 11. Left: distribution of photometric redshifts, $z_{\text {phot }}$, calculated using HyperZ based on the original, positional-matched source catalog in the square-degree test region of XMM-LSS. Details on the calculation of the photometric redshifts are provided in Pforr et al. (2017, in preparation). Only the 24,273 sources with measurements available in all 12 bands and accurate $\left(\chi_{\text {red }}^{2} \lesssim 3.0\right)$ photometric redshifts are included. The inset axis shows the distribution of the 9 sources with high redshifts in the range $4<z_{\text {phot }}<6$. Right: same as the left panel, except here sources with accurate photometric measurements in all 12 bands from our new forced photometric catalog based on The Tractor. Here, a total of 52,166 sources meet the criteria and are shown on the main axis. The inset axis highlights the distribution of 70 sources with high redshifts.

in our square-degree test region, such as stellar mass, for which significantly more accurate measurements can now be made.

For the IRAC-selected input catalog, we found a dramatic improvement in the fraction of red objects with multi-band detections (from $0 \%$ to $69 \%$ at $K_{\mathrm{s}}$-band) in the VIDEO/ CFHTLS-D1 data that were previously identified only in the SERVS imaging. This opens up exciting new prospects for multi-wavelength analyses-including photometric redshift estimates-for large samples of extremely dust-enshrouded galaxies at high redshift.

In the future, we plan to apply our implementation of multiband forced photometry to the full SERVS footprint as well as to new post-cryogenic Spitzer surveys such as DEEPDRILL that are currently in progress. The clear improvement in photometric redshift accuracy that we have demonstrated will ultimately allow us to robustly address a number of key science topics in field of galaxy evolution, including stellar mass assembly, the fraction of obscured star formation, massive black hole growth and feedback, and the role of environment as galaxies grow and evolve.

We thank the referee for providing us with thoughtful comments that have significantly improved the quality of this work. We also thank Scott Ransom for assisting us with the implementation of parallelization in our PYTHON driver script. The National Radio Astronomy Observatory is a facility of the National Science Foundation operated under cooperative agreement by Associated Universities, Inc. G.W. acknowledges financial support for this work from NSF grant AST-1517863 and from NASA through programs GO-13306, GO-13677, GO-13747, \& GO-13845/14327 from the Space Telescope
Science Institute, which is operated by AURA, Inc., under NASA contract NAS 5-26555. M.V. acknowledges support from the European Commission Research Executive Agency (FP7-SPACE-2013-1 GA 607254), the South African Department of Science and Technology (DST/CON 0134/2014), and the Italian Ministry for Foreign Affairs and International Cooperation (PGR GA ZA14GR02).

This work is based on observations made with the Spitzer Space Telescope, which is operated by the Jet Propulsion Laboratory, California Institute of Technology under a contract with NASA. Support for this work was provided by the grant associated with Spitzer proposal 11086. Our analysis includes observations obtained with the MegaPrime/MegaCam instrument, a joint project of CFHT and CEA/IRFU, at the CanadaFrance-Hawaii Telescope (CFHT), which is operated by the National Research Council (NRC) of Canada, the Institut National des Science de l'Univers of the Centre National de la Recherche Scientifique (CNRS) of France, and the University of Hawaii. This study is also based in part on data products produced at Terapix available at the Canadian Astronomy Data Centre as part of the Canada-France-Hawaii Telescope Legacy Survey, a collaborative project of NRC and CNRS. We additionally utilized data from the VIMOS VLT Deep Survey, obtained from the VVDS database operated by Cesam, Laboratoire d'Astrophysique de Marseille, France.

The authors have made use of ASTROPY, a communitydeveloped core PYTHON package for Astronomy (Astropy Collaboration et al. 2013). We also used MONTAGE, which is funded by the National Science Foundation under grant No. ACI-1440620, and was previously funded by the National Aeronautics and Space Administration's Earth Science Technology Office, Computation Technologies Project, under 

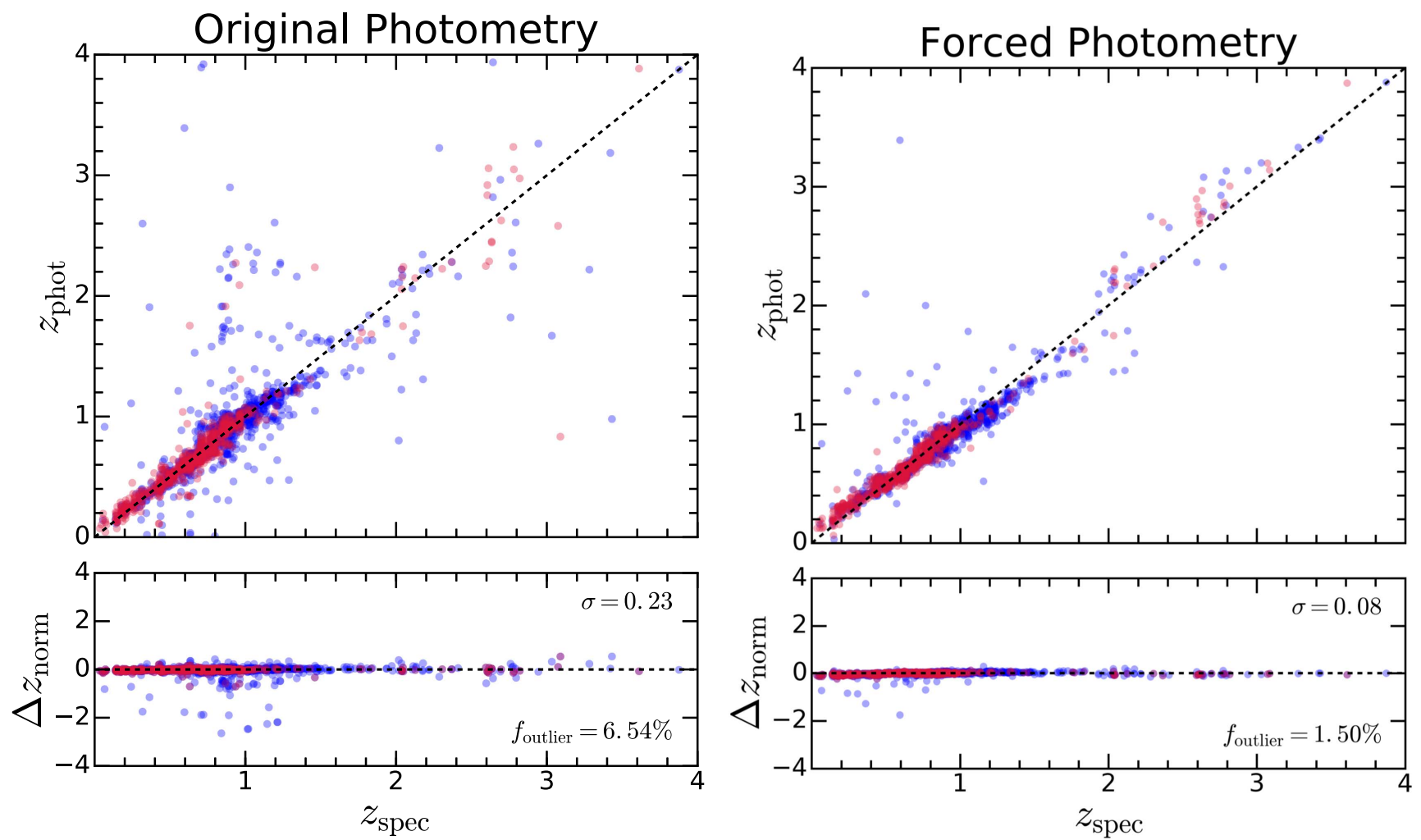

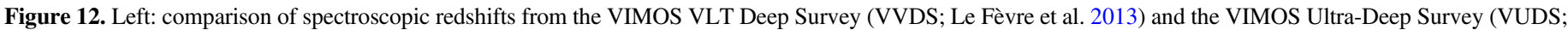

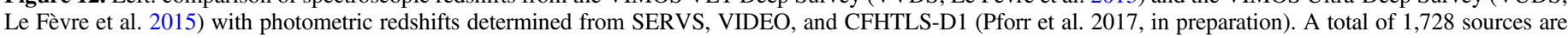

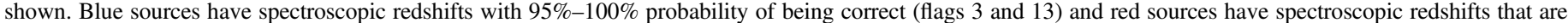

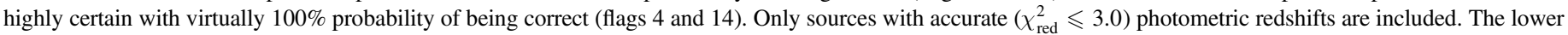

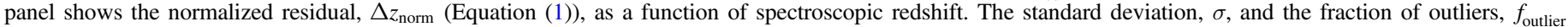

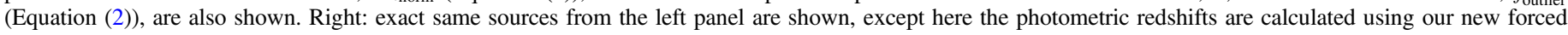
photometry.

Cooperative Agreement Number NCC5-626 between NASA and the California Institute of Technology.

Facilities: Spitzer (IRAC), VISTA, CFHT.

\section{Appendix \\ Photometric Errors}

\section{A.1. SERVS}

Photometric errors for the SERVS bands are estimated as follows:

$$
\sigma_{\text {source }}=3 \times \sqrt{A \sigma_{\mathrm{rms}}^{2}+F / G},
$$

where the factor of 3 approximately accounts for correlated noise between pixels ${ }^{22}, A=\pi r^{2}$ is the circular source area in pixels, $\sigma_{\text {rms }}$ is the background rms noise value per pixel, $F$ is the source brightness in native image units $\left(\mathrm{MJy} \mathrm{sr}^{-1}\right)$, and $G$ is the IRAC weighted gain. For all SERVS sources in The Tractor catalog drawn from the IRAC-selected input catalog, as well as unresolved sources in the VIDEO-selected input catalog, the source radius, $r$, in pixels is determined by $r_{\text {unresolved }}$,

$$
r_{\text {unresolved }}=\sqrt{\Sigma w_{i} \sigma_{i}^{2}}
$$

\footnotetext{
22 The constant value of 3 in Equation (3) encompasses various effects related to the presence of correlated noise between pixels, including the fact that we have down-sampled the native pixel size by factor of two from 1 ." 2 to 0 . 6 and performed drizzling to form the final SERVS irac mosaics.
}

where $w_{i}$ and $\sigma_{i}$ are the $i$ th Gaussian weight and standard deviation parameters that characterize the composite Gaussian PSF models of each band described in Table 2. For resolved sources drawn from the VIDEO-selected input catalog, the source radius, $r$, is instead measured by

$$
r_{\text {resolved }}=\sqrt{2 \times r_{\text {source }}^{2}+r_{\text {unresolved }}^{2}}
$$

where $r_{\text {source }}$ is based on the source radius definition given in Section 3.4 and the factor of two accounts for the fact that $r_{\text {source }}$ traces the half-light radius rather than the full radius.

For $\sigma_{\text {rms }}$, or the sky noise, we measure a single value for each image of each band (see Table 2), and convert from MJy sr${ }^{-1}$ to $\mu \mathrm{Jy}$ per pixel $\left(1 \mathrm{MJy} \mathrm{sr}^{-1} \approx 8.46 \mu \mathrm{Jy} \mathrm{pixel}^{-2}\right.$ ) before inserting this term into Equation (3). The weighted gain, $G$, is defined $^{23}$ by

$$
G=\frac{N \times g \times T}{K}
$$

where $N$ is the average number of coverages estimated from each Spitzer Astronomical Observation Request (AOR; the $X M M$-LSS SERVS observations consisted of 12 AORs), $g$ is the detector gain $\left(3.70 e^{-1}(\mathrm{DN})^{-1}\right.$ for the $3.6 \mu \mathrm{m}$ band and $3.71 e^{-1}(\mathrm{DN})^{-1}$ for the $4.5 \mu \mathrm{m}$ band), $T$ is the exposure time per coverage (100 seconds for SERVS), and $K$ is the

\footnotetext{
${ }^{23}$ For further details on the derivation of the weighted gain, $G$, we refer readers to Section 5.2 of Timlin et al. (2016).
} 
conversion factor from digital to physical units. The flux uncertainty for each source is converted from $\mathrm{MJy} \mathrm{sr}^{-1}$ to $\mu \mathrm{Jy}$ pixel $^{-2}$ using the relation $F\left(\mu \mathrm{Jy}\right.$ pixel $\left.^{-2}\right)=8.46 \times \mathrm{F}$ $\left(\mathrm{MJy} \mathrm{sr}^{-1}\right)$.

\section{A.2. VIDEO and CFHTLS-D1}

For the ground-based VIDEO and CFHTLS-D1 bands, we estimate the photometric errors according to

$$
\sigma_{\text {source }}=C \times \sqrt{A \sigma_{\mathrm{rms}}^{2}+(0.03 \times F)^{2}},
$$

where $A$ and $\sigma_{\text {rms }}^{2}$ are defined as in Equation (3), $F$ is the source flux in $\mu \mathrm{Jy}$, and the factor of 0.03 accounts for the expected photometric error due to systematic uncertainties in the underlying image calibration. The constant $C$ in Equation A5 accounts for correlated pixel noise, and has values of 3 for VIDEO and 1 for CFHTLS-D1.

\section{A.3. Magnitude Uncertainties}

Magnitude uncertainties for all bands are calculated following the standard conversion from the flux uncertainties using

$$
\sigma_{\text {mag }}=1.089 \times \sigma_{\text {source }} / f_{\text {source }},
$$

where $f_{\text {source }}$ is the source brightness and both $\sigma_{\text {source }}$ and $f_{\text {source }}$ are measured in units of $\mu \mathrm{Jy}$.

\section{References}

Aihara, H., Armstrong, R., Bickerton, S., et al. 2017, arXiv:1702.08449 Astropy Collaboration, Robitaille, T. P., Tollerud, E. J., et al. 2013, A\&A, 558, A33

Berriman, G. B., \& Good, J. C. 2017, PASP, 975, 058006

Bertin, E. 2011, in ASP Conf. Ser. 442, Astronomical Data Analysis Software and Systems XX, ed. I. N. Evans et al. (San Francisco, CA: ASP), 435

Bertin, E., \& Arnouts, S. 1996, A\&AS, 117, 393

Bolzonella, M., Miralles, J.-M., \& Pelló, R. 2000, A\&A, 363, 476

Budavári, T., \& Basu, A. 2016, AJ, 152, 86

Dark Energy Survey Collaboration, Abbott, T., Abdalla, F. B., et al. 2016, MNRAS, 460, 1270

Fazio, G. G., Hora, J. L., Allen, L. E., et al. 2004, ApJS, 154, 10

Feigelson, E. D., \& Babu, G. J. 2012, Modern Statistical Methods for Astronomy (Cambridge: Cambridge Univ. Press)
Flewelling, H. A., Magnier, E. A., Chambers, K. C., et al. 2016, arXiv:1612. 05243

Gwyn, S. D. J. 2012, AJ, 143, 38

Hönig, S. F., Watson, D., Kishimoto, M., et al. 2017, MNRAS, 464, 1693

Hora, J. L., Marengo, M., Park, R., et al. 2012, Proc. SPIE, 8442, 844239

Huber, M. \& PS1-IPP Team, P.S.C. 2015, in American Astronomical Society Meeting Abstracts, \#225, 336.17

Hurley, P. D., Oliver, S., Betancourt, M., et al. 2017, MNRAS, 464, 885

Ilbert, O., Capak, P., Salvato, M., et al. 2009, ApJ, 690, 1236

Jarvis, M. J., Bonfield, D. G., Bruce, V. A., et al. 2013, MNRAS, 428, 1281

Kron, R. G. 1980, ApJS, 43, 305

Lang, D., Hogg, D. W., \& Mykytyn, D. 2016a, The Tractor: Probabilistic Astronomical Source Detection and Measurement, Astrophysics Source Code Library, ascl:1604.008

Lang, D., Hogg, D. W., \& Schlegel, D. J. 2016b, AJ, 151, 36

Lawrence, A., Warren, S. J., Almaini, O., et al. 2007, MNRAS, 379, 1599

Le Fèvre, O., Cassata, P., Cucciati, O., et al. 2013, A\&A, 559, A14

Le Fèvre, O., Tasca, L. A. M., Cassata, P., et al. 2015, A\&A, 576, A79

Lonsdale, C. J., Smith, H. E., Rowan-Robinson, M., et al. 2003, PASP, 115,897

Mancone, C. L., Gonzalez, A. H., Moustakas, L. A., \& Price, A. 2013, PASP, 125,1514

Maraston, C. 2005, MNRAS, 362, 799

Marquez, M. J., Budavári, T., \& Sarro, L. M. 2014, A\&A, 563, A14

Mauduit, J.-C., Lacy, M., Farrah, D., et al. 2012, PASP, 124, 714

Merlin, E., Amorín, R., Castellano, M., et al. 2016a, A\&A, 590, A30

Merlin, E., Bourne, N., Castellano, M., et al. 2016b, A\&A, 595, A97

Merlin, E., Fontana, A., Ferguson, H. C., et al. 2015, A\&A, 582, A15

Oesch, P. A., Bouwens, R. J., Illingworth, G. D., et al. 2014, ApJ, 786, 108

Oliver, S. J., Bock, J., Altieri, B., et al. 2012, MNRAS, 424, 1614

Papovich, C., Shipley, H. V., Mehrtens, N., et al. 2016, ApJS, 224, 28

Peng, C. Y., Ho, L. C., Impey, C. D., \& Rix, H.-W. 2002, AJ, 124, 266

Pforr, J., Maraston, C., \& Tonini, C. 2013, MNRAS, 435, 1389

Richards, G. T., Myers, A. D., Peters, C. M., et al. 2015, ApJS, 219, 39

Steidel, C. C., Adelberger, K. L., Shapley, A. E., et al. 2003, ApJ, 592, 728

Steidel, C. C., Hunt, M. P., Shapley, A. E., et al. 2002, ApJ, 576, 653

Taylor, M. B. 2005, in ASP Conf. Ser. 347, Astronomical Data Analysis Software and Systems XIV, ed. P. Shopbell, M. Britton, \& R. Ebert (San Francisco, CA: ASP), 29

Timlin, J. D., Ross, N. P., Richards, G. T., et al. 2016, ApJS, 225, 1

Vaccari, M. 2015, in The Many Facets of Extragalactic Radio Surveys: Towards New Scientific Challenges, Galaxy Evolution I (Trieste: SISSA), 27

Vaccari, M., Covone, G., Radovich, M., et al. 2017, arXiv:1704.01495

Wang, W.-H., Barger, A. J., \& Cowie, L. L. 2012, ApJ, 744, 155

Werner, M. W., Roellig, T. L., Low, F. J., et al. 2004, ApJS, 154, 1

Wright, A. H., Robotham, A. S. G., Bourne, N., et al. 2016, MNRAS, 460, 765

Yan, H., Dickinson, M., Eisenhardt, P. R. M., et al. 2004, ApJ, 616, 63 